\title{
Insect galls of Itamonte (Minas Gerais, Brazil): characterization and occurrence
}

\author{
Valéria Cid Maia ${ }^{1}$ \\ ${ }^{I}$ Departamento de Entomologia, Museu Nacional, Quinta da Boa Vista, São Cristóvão, CEP \\ 20940-040 Rio de Janeiro, RJ, Brazil,e-mail:maiavcid@acd.ufri.br \\ Corresponding author: Valéria Cid Maia,e-mail: maiavcid@acd.ufri.br
}

MAIA, V. C. Insect galls of Itamonte (Minas Gerais, Brazil): characterization and occurrence. Biota Neotropica. 14(1): e20133839. http://www.biotaneotropica.org.br/v14n1/en/abstract?article+bn01114012014

\begin{abstract}
Three sites of Itamonte (Minas Gerais) were investigated for insect galls from September, 2011 to July, 2012, seasonally. One-hundred and one morphotypes of insect galls were recorded on 63 species of host plant (45 genera and 23 families). Melastomataceae was the most galled plant family, followed by Asteraceae and Myrtaceae. Galls were recorded on leaves, stems, buds, and aerial roots. Leaves were the most galled plant organ. The gall morphotypes were characterized according to their shape, color, pubescence, and number of internal chambers. Globose and fusiform galls were the most frequent. The majority was glabrous and one-chambered. The gallers comprised three insect orders: Diptera (Cecidomyiidae and Tephritidae), Hemiptera, and Lepidoptera. Cecidomyiidae were the most frequent galling taxa. Parasitoids (Hymenoptera) were obtained from six morphotypes (ca. 6\% of the total). The results are compared to those of other Brazilian insect gall inventories. As there is no previous gall inventory in Itamonte, all records presented here are new.
\end{abstract}

Keywords: Atlantic forest, galling insect, gall richness, insect-plant interaction.

MAIA, V. C. Galhas de insetos de Itamonte (Minas Gerais, Brasil): caracterização e ocorrência. Biota Neotropica. 14(1):e20133839. http://www.biotaneotropica.org.br/v14n1/pt/abstract?article+bn01114012014

Resumo - Três localidades de Itamonte (Minas Gerais) foram investigadas para galhas de inseto no período de setembro, 2011 a julho, 2012, sazonalmente. Cento e um morfotipos de galhas de inseto foram registrados em 63 espécies de plantas hospedeiras (45 gêneros e 23 famílias). Melastomataceae, Asteraceae e Myrtaceae foram as famílias de planta com maior riqueza de galhas. As galhas foram encontradas em folhas, caules, gemas e raízes aéreas. As folhas foram o órgão vegetal com maior incidência de galhas. Os morfotipos foram caracterizados em relação à forma, coloração, pubescência e número de câmaras internas. Galhas globosas e fusiformes foram as mais frequentes. A maioria era glabra e unilocular. Os galhadores incluíram três ordens de insetos: Diptera (Cecidomyiidae and Tephritidae), Hemiptera e Lepidoptera. Os Cecidomyiidae foram o táxon galhador mais frequente. Parasitóides (Hymenoptera) foram obtidos de seis morfotipos de galhas (cerca de 6\% do total). Os resultados são comparados com outros inventários de galhas no Brasil. Como não havia inventário prévio de galhas em Itamonte, todos os registros apresentados são novos.

Palavras-chave: Mata Atlântica, inseto galhador, riqueza de galhas, interação inseto-planta.

\section{Introduction}

Galls are abnormal growths of plant cells, tissues or organs induced by virus, bacteria, nematodes, mites, insects, and other organisms. Among the insects, six orders comprise galling species: Diptera, Hemiptera, Coleoptera, Lepidoptera, Hymenoptera, and Thysanoptera (Felt 1940). Galling insects are considered as one of the most specialized herbivores, showing high specificity of host plant and plant organ (Carneiro et al. 2009). Each galling species induces a single gall morphotype on the host plant, so the gall is considered as a taxonomic character of the galler.

The galling insect fauna of Brazil is still little studied. Data are restricted to few localities and biomes, mainly restinga areas in the State of Rio de Janeiro (Monteiro et al. 1994, Maia 2001, Monteiro et al. 2004, Oliveira \& Maia 2005, Maia \& Oliveira 2010), São Paulo (Maia et al. 2008) and Espírito Santo (Bregonci et al. 2010), and in cerrado areas of the State of Minas Gerais (Fernandes et al. 1988, Fernandes et al. 1997; Urso-Guimarães et al. 2003, Maia \& Fernandes 2004, Carneiro et al. 2009, Coelho et al. 2009), São Paulo (Urso-Guimarães \& Scareli-Santos 2006, Saito \& Urso-Guimarães 2012) and Goiás (Araújo et al. 2007). The taxonomical information of the gallers is quite incipient, as the majority of the records are at family level.

The main objective of this work is to contribute to the knowledge of the insect galls of Minas Gerais, investigating remaining areas of Atlantic forest as well high altitude fields for the first time in the Municipality of Itamonte. 


\section{Material and Methods}

The survey was developed in Itamonte (Minas Gerais, Southeastern Brazil). The municipality of Itamonte has an area of about $432 \mathrm{~km}^{2}$. The climate is highland tropical (Cwb), according to Köppen classification, with cold and dry winters (lowest temperature varying from $0^{\circ} \mathrm{C}$ to $10^{\circ} \mathrm{C}$ in the urban area with the occurrence of frosts); mild summer with higher rainfall, characterizing the rainiest period (Itamonte 2012).

Itamonte has about $35 \%$ of the area of the Parque Nacional de Itatiaia (Itatiaia National Park). This Park has about 30,000 ha and is divided into lower and upper areas. The former has remainings of the Atlantic Forest, with great richness of orchids, bromeliads and ferns. The latter presents low vegetation and predominance of high altitude fields. The Itatiaia massif comprises about 150 endemic plant species, more than 100,000 insect species and 300 bird species (Zikan \& Zikan 1968, Martinelli et al. 1989, Honkala \& Niiranen 2010, Itamonte 2012).

Insect galls were collected from September, 2011 to July, 2012, at the end of spring, summer, autumn, and winter, in three localities: Brejo da Lapa $\left(22^{\circ} 21^{\prime} 31^{\prime \prime} \mathrm{S}, 44^{\circ} 44^{\prime} 14^{\prime \prime} \mathrm{W}\right.$; $2,100 \mathrm{~m}$ of altitude, low vegetation); Cachoeira do Escorrega $\left(22^{\circ} 13^{\prime} 13,75^{\prime \prime} \mathrm{S}, 44^{\circ} 49^{\prime} 16,05^{\prime \prime} \mathrm{W} ; 1,717 \mathrm{~m}\right.$ of altitude, Atlantic Forest), and Sítio Moana $\left(22^{\circ} 14^{\prime} 23^{\prime \prime} \mathrm{S}, 44^{\circ} 47^{\prime} 55^{\prime \prime} \mathrm{W} ; 1320 \mathrm{~m}\right.$ of altitude, Atlantic Forest).

The vegetation of each locality was investigated for insect galls for 4 hours per visit. All plant organs were examined, except for the subterraneous roots. Samples of host plants, preferably in the fertile state, were pressed in the field and later identified by Dr. Gracialda C. Ferreira and Manoel dos Reis
Cordeiro (Universidade Federal Rural da Amazônia, Pará, northern Brazil). The voucher specimens were included in the Herbarium of this Institution.

All gall morphotypes were photographed in the field. Each morphotype was characterized based on shape, color, presence or absence of trichomes, and number of internal chambers. Immature insects were obtained by dissection of each kind of gall under a stereoscopic microscope. Pupal exuviae and adults were obtained by keeping samples of each gall morphotype individually in plastic pots layered at the bottom with damp cotton and covered by fine screening, at room temperature. All pots were checked daily for adult emergence.

All insects were preserved in 70\% alcohol. Cecidomyidae (Diptera) were later mounted on slides for microscope, following the methodology of Gagné (1994). The gall midge genera were identified based on the keys by Gagné (1994). The specimens are deposited in the collection of Museu Nacional/ Universidade Federal do Rio de Janeiro.

\section{Results}

One hundred and one morphotypes of insect galls were found on 63 species of host plant (45 genera and 23 families) (Table 1). Melastomataceae is the most galled plant family, followed by Asteraceae and Myrtaceae, with 29, 18 and 11 gall morphotypes, respectively. The average number of galls per host species was 1.60 .

Galls were recorded on leaves, stems, buds, and aerial roots (Table 2). Leaves were the most galled plant organ, with ca. $48 \%$ of the total, followed by stems and buds $(29 \%$ and $21 \%$, respectively). Otherwise, galls on tendrils, flowers, and fruits

Table 1. Number of gall morphotypes per plant family in Itamonte (MG, Brazil). Tabela 1. Número de morfotipos de galha por família de planta em Itamonte (MG, Brasil)

\section{Host plant}

\begin{tabular}{|c|c|c|c|}
\hline Galled families $(n=23)$ & $\begin{array}{l}\text { Number of galled genera } \\
\qquad(n=45)\end{array}$ & $\begin{array}{l}\text { Number of galled species } \\
\qquad(n=63)\end{array}$ & $\begin{array}{l}\text { Number of gall morphotypes } \\
\qquad(n=101)\end{array}$ \\
\hline Acanthaceae & 1 & 1 & 1 \\
\hline Apocynaceae & 1 & 1 & 1 \\
\hline Asteraceae & 6 & 10 & 18 \\
\hline Bignoniaceae & 3 & 3 & 3 \\
\hline Burseraceae & 1 & 1 & 1 \\
\hline Campanulaceae & 1 & 1 & 1 \\
\hline Euphorbiaceae & 1 & 2 & 5 \\
\hline Fabaceae & 2 & 2 & 2 \\
\hline Iracinaceae & 1 & 1 & 1 \\
\hline Lammiaceae & 1 & 1 & 1 \\
\hline Lauraceae & 3 & 3 & 3 \\
\hline Loranthaceae & 1 & 1 & 4 \\
\hline Lythraceae & 1 & 1 & 1 \\
\hline Melastomataceae & 6 & 15 & 29 \\
\hline Myrtaceae & 4 & 7 & 11 \\
\hline Nyctaginaceae & 1 & 1 & 1 \\
\hline Piperaceae & 1 & 1 & 1 \\
\hline Proteaceae & 1 & 1 & 2 \\
\hline Rubiaceae & 4 & 4 & 4 \\
\hline Salicaceae & 2 & 2 & 4 \\
\hline Sapindaceae & 1 & 1 & 1 \\
\hline Solanaceae & 1 & 2 & 3 \\
\hline Vochysiaceae & 1 & 1 & 1 \\
\hline
\end{tabular}


Table 2. Number of gall morphotypes per plant organ in Itamonte (MG, Brazil). Tabela 2. Número de morfotipo de galhas por órgão vegetal em Itamonte (MG, Brasil).

\begin{tabular}{lc}
\hline Galled plant organ & Number of gall morphotypes \\
\hline Leaf & $52(48.1 \%)$ \\
Stem & $31(28.7 \%)$ \\
Bud & $23(21.3 \%)$ \\
Aerial root & $2(1.85 \%)$ \\
Tendril & 0 \\
Inflorescence & 0 \\
Fruit & 0 \\
\hline
\end{tabular}

were not found. All morphotypes occurred in a single plant organ, except seven morphotypes which occurred simultaneously on stem and leaf $(n=3)$, stem and bud $(n=2)$, and bud and leaf $(n=2)$.

The gall morphotypes were classified according to their shape in globose, fusiform, circular, marginal roll, ovoid, leaf roll, leaf fold, and rosette. The globose and fusiform shapes were the most frequent ones (about 38\% each), followed by marginal roll (about $8 \%$ ), circular and conical (each about 7\%), ovoid (about $2 \%$ ), and others (each at about 1\%) (Table 3 ). The majority was glabrous (81 morphotypes, ca. 80\%), the remainders being (20 morphotypes, ca. 20) hairy. About $97 \%$ (98 morphotypes) are one-chambered, with 3\% (four morphotypes) multichambered.

The gallers of 65 morphotypes were determined. They belong to three insect orders: Diptera (Cecidomyiidae and Tephritidae), Hemiptera, and Lepidoptera. The former was the most frequent galling taxa, being responsible for 51 morphotypes ( $78 \%$ of the determined gallers) (Table 4$)$, followed by Hemiptera (ca. 12\%), and Lepidoptera (ca. 9\%). Coleopterous, Hymenopterous and Thysanopterous galls were not found. The gallers of 36 morphotypes cannot be determined, because the

Table 3. Number of gall morphotypes of different shapes in Itamonte (MG, Brazil). Tabela 3. Número de morfotipos de galha de diferentes formas em Itamonte (MG, Brasil).

\begin{tabular}{lc}
\hline Gall shape & Number of gall morphotypes \\
\hline Fusiform & $38(37.62 \%)$ \\
Globose & $38(37.62 \%)$ \\
Marginal roll & $8(7.92 \%)$ \\
Circular & $7(6.93 \%)$ \\
Conical & $7(6.93 \%)$ \\
Ovoid & $2(1.98 \%)$ \\
Rosette & $1(0.99 \%)$ \\
Leaf fold & $1(0.99 \%)$ \\
Leaf roll & $1(0.99 \%)$ \\
\hline
\end{tabular}

Table 4. Number of gall morphotypes per galling insect order in Itamonte (MG, Brazil). Tabela 4. Número de morfotipos de galha por ordem de inseto galhador em Itamonte (MG, Brazil).

\begin{tabular}{lc}
\hline Galler & Number of gall morphotypes \\
\hline Diptera & $51(78.5 \%)$ \\
Hemiptera & $(12.3 \%)$ \\
Lepidoptera & $(9.2 \%)$ \\
Not determined & $(35.6 \%)$ \\
\hline
\end{tabular}

gall samples were collected without dwellers, or less frequently, occupied by parasitoids (in this case, fragments of the host were found). Parasitoids (Hymenoptera) were obtained from six morphotypes (ca. $6 \%$ of the total). Predators and inquilines were not found. As successors, ants were found in a single gall morphotype.

As there is no previous gall inventory in Itamonte, all records presented here are new. The geographic distribution of Lopesia similis Maia, 2004, and Neolasioptera eugeniae Maia, 1993 (Diptera, Cecidomyiidae) is widened to the municipality of Itamonte.

Data on insect galls are presented here under host plant families, genera and species in alphabetical order. They include gall morphological characterization, associated fauna and previous records in Brazil.

\section{Acanthaceae}

Not determined

Vein or petiole swelling, fusiform, green, glabrous, onechambered (Figures 1 and 2). Galler: Cecidomyiidae (Diptera). Locality: Cachoeira do Escorrega. Occurrence: March. Previous records: Galls on Justicia sp. and Avicennia shaeuriana Stapf \& Leechm. ex Moldenke (Acanthaceae) were recorded in Rio Grande do Sul and São Paulo, respectively by Tavares (1909) and Maia et al. (2008).

\section{Apocynaceae}

\section{Aspidosperma sp.}

Circular leaf gall, green, glabrous, one-chambered (Figure 3). Galler: Psyliidae (Hemiptera). Locality: Cachoeira do Escorrega. Occurrence: June.

Previous records: leaf galls on Aspidosperma spp. were recorded in the States of Goiás, Mato Grosso do Sul, Minas Gerais, and Rio de Janeiro (Fernandes et al. 1988, Monteiro et al. 1994, Maia 2001a, Julião et al. 2002, Oliveira \& Maia 2005, Araujo et al. 2007, Coelho et al. 2009, Maia \& Oliveira 2010).

\section{Asteraceae}

\section{Baccharis sp. 1}

Vein swelling, fusiform, green, glabrous, one-chambered. Galler: Cecidomyiidae (Diptera). Associated fauna: parasitoid Hymenoptera. Locality: Brejo da Lapa. Occurrence: December.

Baccharis sp.2

Leaf gall, fusiform, green, glabrous, one-chambered (Figure 4). Galler: Cecidomyiidae (Diptera). Locality: Brejo da Lapa. Occurrence: September.

Previous records: Several galls on Baccharis spp. have been recorded by in cerrado and rupestrian fields of Minas Gerais by Fernandes et al. (1996), Maia \& Fernandes (2004), Coelho et al. (2009), Carneiro et al. (2009), Malves \& Frieiro-Costa (2012), and in restingas of São Paulo by Maia et al. (2008).

\section{Eupatorium sp.}

Bud gall, globose, brownish, micropubescent, one-chambered (Figure 5). Galler: Cecidomyiidae (Diptera). Associated fauna: parasitoid - Hymenoptera. Locality: Brejo da Lapa. Occurrence: September.

Previous records: Galls on Eupatorium spp. were recorded by Tavares (1917b) in Rio de Janeiro.

\section{Mikania sp.1}

Stem swelling, globose, brownish, glabrous, one-chambered (Figure 6). Galler: Cecidomyiidae (Diptera). Locality: Brejo da Lapa. Occurrence: June. 

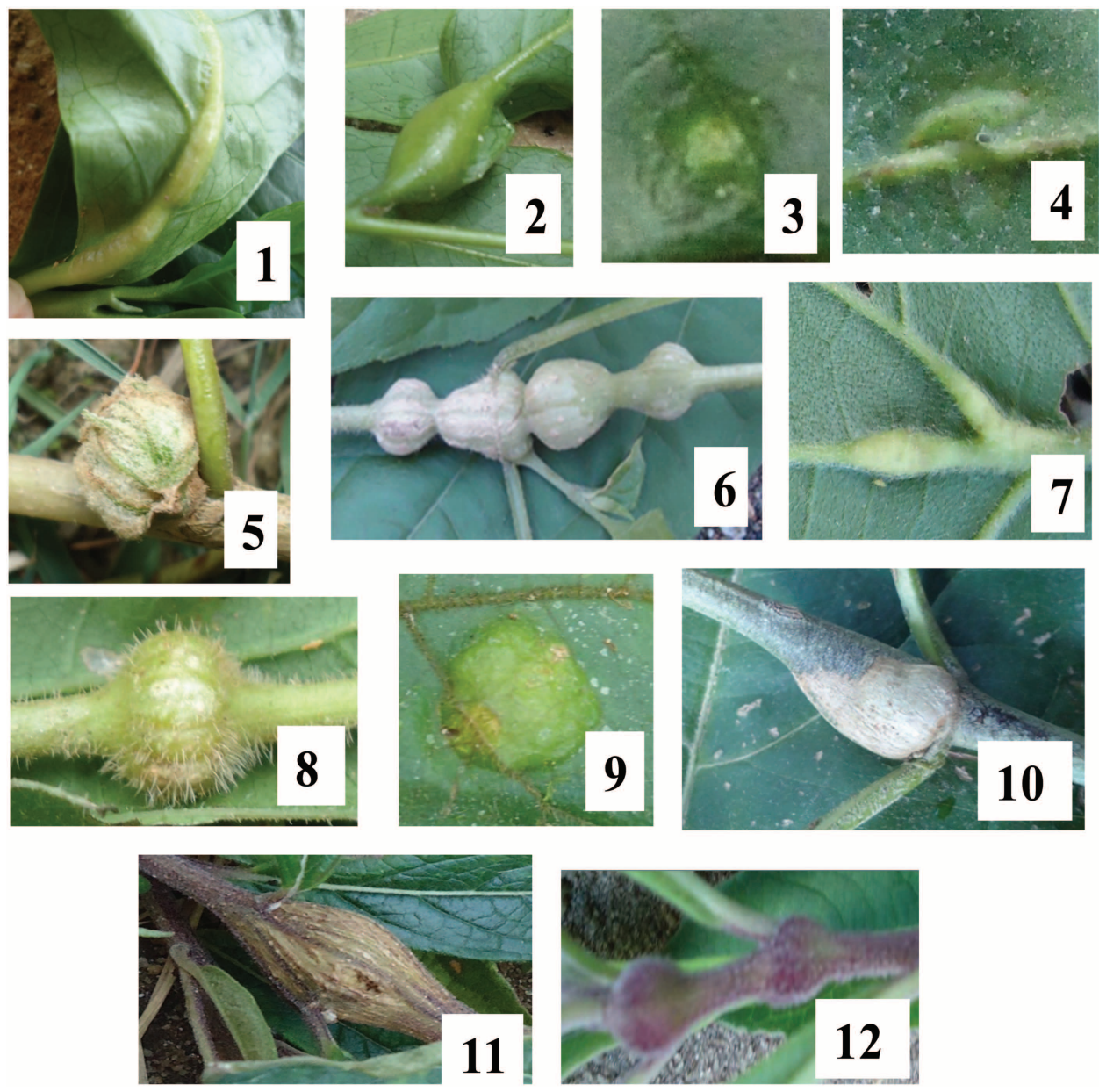

Figures 1-12. Insect galls of Itamonte (MG, Brazil): 1. Vein gall on Acanthaceae; 2. Petiole gall on Acanthaceae not determined; 3. Leaf gall on Aspidosperma sp. (Apocynaceae); 4. Leaf gall on Baccharis sp.2 (Asteraceae); 5. Bud gall on Eupatorium sp. (Asteraceae); 6. Stem gall on Mikania sp.1 (Asteraceae); 7. Vein gall on Mikania sp.1 (Asteraceae); 8. Stem gall on Mikania sp.2 (Asteraceae); 9. Leaf gall on Mikania sp.3 (Asteraceae); 10. Stem gall on Vernonia sp.1 (Asteraceae); 11. Stem gall on Vernonia sp.2 (Asteraceae); 12. Stem gall on Vernonia sp.2 (Asteraceae).Figuras 1-12. Galhas de insetos de Itamonte (MG, Brasil): 1. Galha na nervura em Acanthaceae; 2. Galha no pecíolo em Acanthaceae não determinada; 3. Galha foliar em Aspidosperma sp. (Apocynaceae); 4. Galha foliar em Baccharis sp.2 (Asteraceae); 5. Galha da gema em Eupatorium sp. (Asteraceae); 6. Galha caulinar em Mikania sp.1 (Asteraceae); 7. Galha na nervura em Mikania sp.1 (Asteraceae); 8. Galha caulinar em Mikania sp.2 (Asteraceae); 9. Galha foliar em Mikania sp.3 (Asteraceae); 10. Galha caulinar em Vernonia sp.1 (Asteraceae); 11. Galha caulinar em Vernonia sp.2 (Asteraceae);12. Galha caulinar em Vernonia sp.2 (Asteraceae).

Vein swelling, fusiform, green, glabrous, one-chambered (Figure 7). Galler: Cecidomyiidae (Diptera). Associated fauna: parasitoid - Hymenoptera. Locality: Brejo da Lapa. Occurrence: September, December

Mikania sp.2

Stem or petiole swelling, globose, green, hairy, onechambered (Figure 8). Galler: not determined. Locality: Brejo da Lapa. Occurrence: September.

Mikania sp.3

Leaf gall, circular, green, glabrous, one-chambered (Figure 9). Galler: Cecidomyiidae (Diptera). Locality: Cachoeira do Escorrega. Occurrence: March.

Previous records: several galls on Mikania spp. were recorded in Amazonas, Minas Gerais, Rio de Janeiro, and Rio Grande do Sul (Rübsaamen 1907, 1908, 1916a, Tavares 1909, Monteiro et al. 1994, Maia 2001a, Maia \& Fernandes 2004, Oliveira \& Maia, 2005, Maia et al. 2008, Carneiro et al. 2009, Maia \& Oliveira 2010, Malves \& FrieiroCosta 2012).
Vernonia sp. 1

Stem or bud swelling, fusiform, greenish, glabrous, onechambered (Figure 10). Galler: Cecidomyiidae (Diptera). Locality: Brejo da Lapa. Occurrence: September.

Vernonia sp. 2

Stem swelling, fusiform, brown, glabrous, one-chambered (Figure 11). Galler: Tephritidae (Diptera). Localities: Sitio Moana, Cachoeira do Escorrega. Occurrence: September, March, June.

Stem swelling, globose, reddish, glabrous, one-chambered (Figure 12). Galler: Cecidomyiidae (Diptera). Locality: Cachoeira do Escorrega. Occurrence: December, March.

Previous records: galls on Vernonia spp. were recorded in Mato Grosso do Sul, Minas Gerais, Rio de Janeiro, and São Paulo (Gagné 1994, Julião et al. 2002, Urso-Guimarães et al. 2003, Maia 2001a, Monteiro et al. 2004, Maia \& Fernandes 2004, Maia et al. 2008).

Asteraceae not determined (sp.1)

Rosette bud gall, green, glabrous (Figure 13). Galler: not 

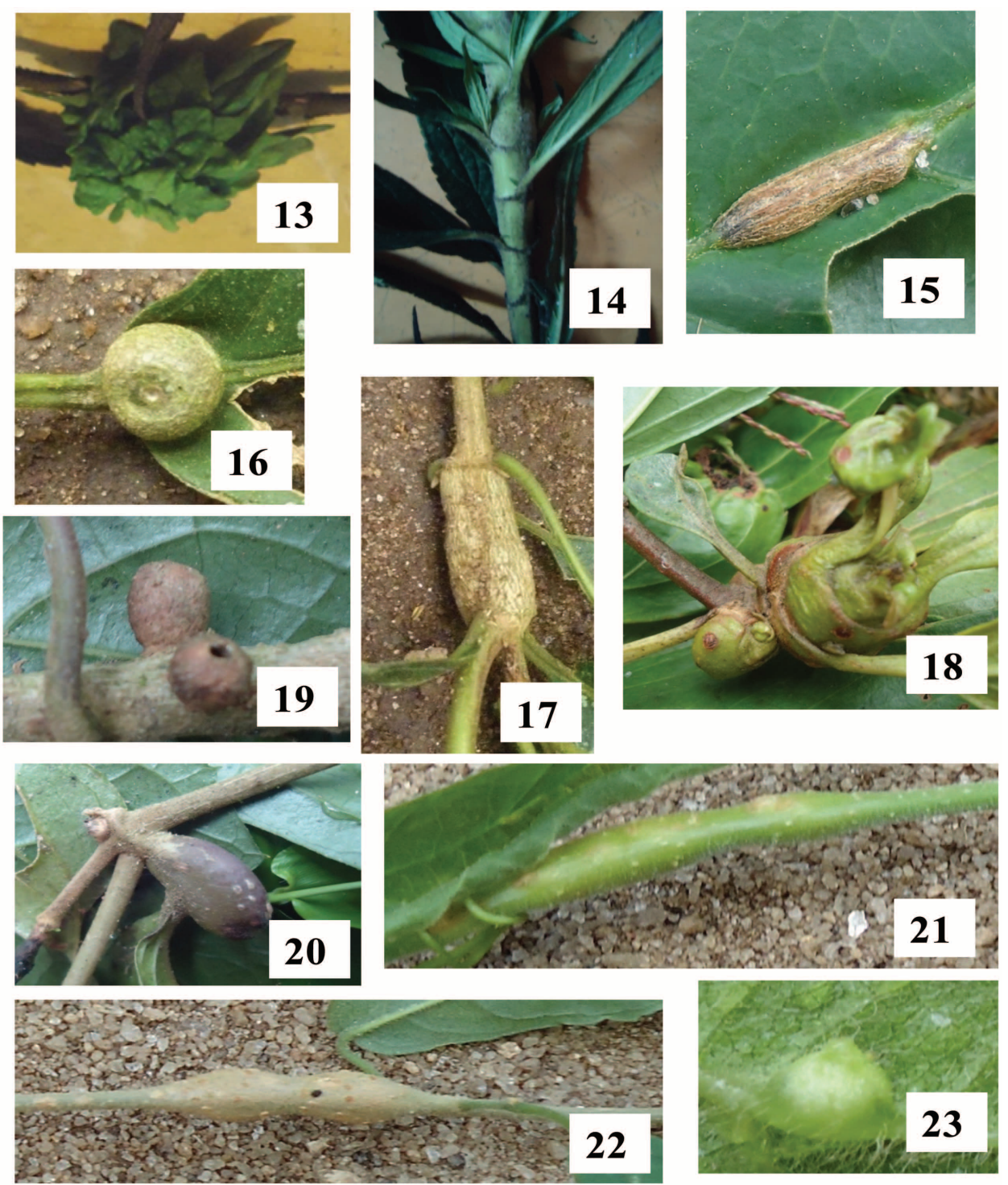

Figures 13-23. Insect galls of Itamonte (MG, Brazil): 13. Bud gall on Asteraceae sp.1; 14. Stem gall on Asteraceae sp.1; 15. Vein gall on Asteraceae sp.2; 16. Petiole gall on Asteraceae sp.2; 17. Stem gall on Asteraceae sp.2; 18. Apical bud gall on Asteraceae sp.2; 19. Lateral bud on Asteraceae sp.2; 20. Lateral bud on Asteraceae sp.2; 21. Stem gall on Cuspidaria sp. (Bignoniaceae); 22. Stem gall on Stizophyllum riparum (Bignoniaceae); 23. Leaf gall on Bignoniaceae.Figuras 13-23. Galhas de insetos de Itamonte (MG, Brasil): 13. Galha da gema em Asteraceae sp.1; 14. Galha caulinar em Asteraceae sp.1; 15. Galha na nervura em Asteraceae sp.2; 16. Galha no pecíolo em Asteraceae sp.2; 17. Galha caulinar em Asteraceae sp.2; 18. Galha da gema apical em Asteraceae sp.2; 19. Galha da gema lateral em Asteraceae sp.2; 20. Galha da gema lateral em Asteraceae sp.2; 21. Galha caulinar em Cuspidaria sp. (Bignoniaceae); 22. Galha caulinar em Stizophyllum riparum (Bignoniaceae); 23. Galha foliar em Bignoniaceae.

determined. Locality: Brejo da Lapa. Occurrence: December

Stem swelling, elongate-fusiform, green, glabrous, onechambered (Figure 14). Galler: Lepidoptera. Locality: Brejo da Lapa. Occurrence: December, June.

Asteraceae not determined (sp. 2)

Vein swelling, linear, brown, glabrous, one-chambered (Figure 15). Galler: Cecidomyiidae (Diptera). Locality: Cachoeira do Escorrega. Occurrence: June.

Petiole swelling, globose, green, glabrous, one-chambered (Figure 16). Galler: not determined. Locality: Cachoeira do Escorrega. Occurrence: March.

Stem swelling, fusiform, brown, glabrous, one-chambered (Figure 17). Galler: not determined. Localities: Brejo da Lapa, Cachoeira do Escorrega. Occurrence: December, March, June.

Apical bud gall, globose, green, glabrous, one-chambered
(Figure 18). Galler: Cecidomyiidae (Diptera). Associated fauna: parasitoid - Hymenoptera. Localities: Brejo da Lapa, Cachoeira do Escorrega. Occurrence: December, March.

Lateral bud gall, globose, brown, glabrous, one-chambered (Figure 19). Galler: Cecidomyiidae (Diptera). Locality: Cachoeira do Escorrega. Occurrence: December.

Lateral bud gall, ovoid, reddish, glabrous, one-chambered (Figure 20). Galler: not determined. Locality: Cachoeira do Escorrega. Occurrence: December.

\section{Bignoniaceae}

Cuspidaria sp.

Stem swelling, elongate-fusiform, green, glabrous, onechambered (Figure 21). Galler: not determined. Locality: Cachoeira do Escorrega. Occurrence: March. 
Stizophyllum riparum (Kunth) Sandwith

Stem swelling, fusiform, brown, glabrous, one-chambered (Figure 22). Galler: not determined. Locality: Cachoeira do Escorrega. Occurrence: March.

Bignoniaceae not determined

Leaf gall, globose, green, hairy, one-chambered (Figure 23). Galler: Cecidomyiidae (Diptera). Locality: Cachoeira do Escorrega. Occurrence: December, June.

\section{Burseraceae}

Protium heptaphyllum March

Marginal leaf roll, green, glabrous, one-chambered (Figure 24). Galler: Lopesia similis Maia, 2004 (Cecidomyiidae, Diptera). Locality: Cachoeira do Escorrega. Occurrence: December, June.
Previous records: the same gall was recorded in Bahia, Minas Gerais, and Rio de Janeiro (Tavares 1922, Maia 2001, Monteiro et al. 2004, Maia \& Fernandes 2004).

\section{Campanulaceae}

Centropogon sp.

Leaf gall, globose abaxially, conical adaxially, green, glabrous, one-chambered (Figure 25). Galler: Hemiptera. Locality: Brejo da Lapa. Occurrence: March.

\section{Euphorbiaceae}

Croton sp.1

Stem swelling, fusiform, green, glabrous, one-chambered (Figure 26). Galler: not determined. Locality: Brejo da Lapa. Occurrence: September.
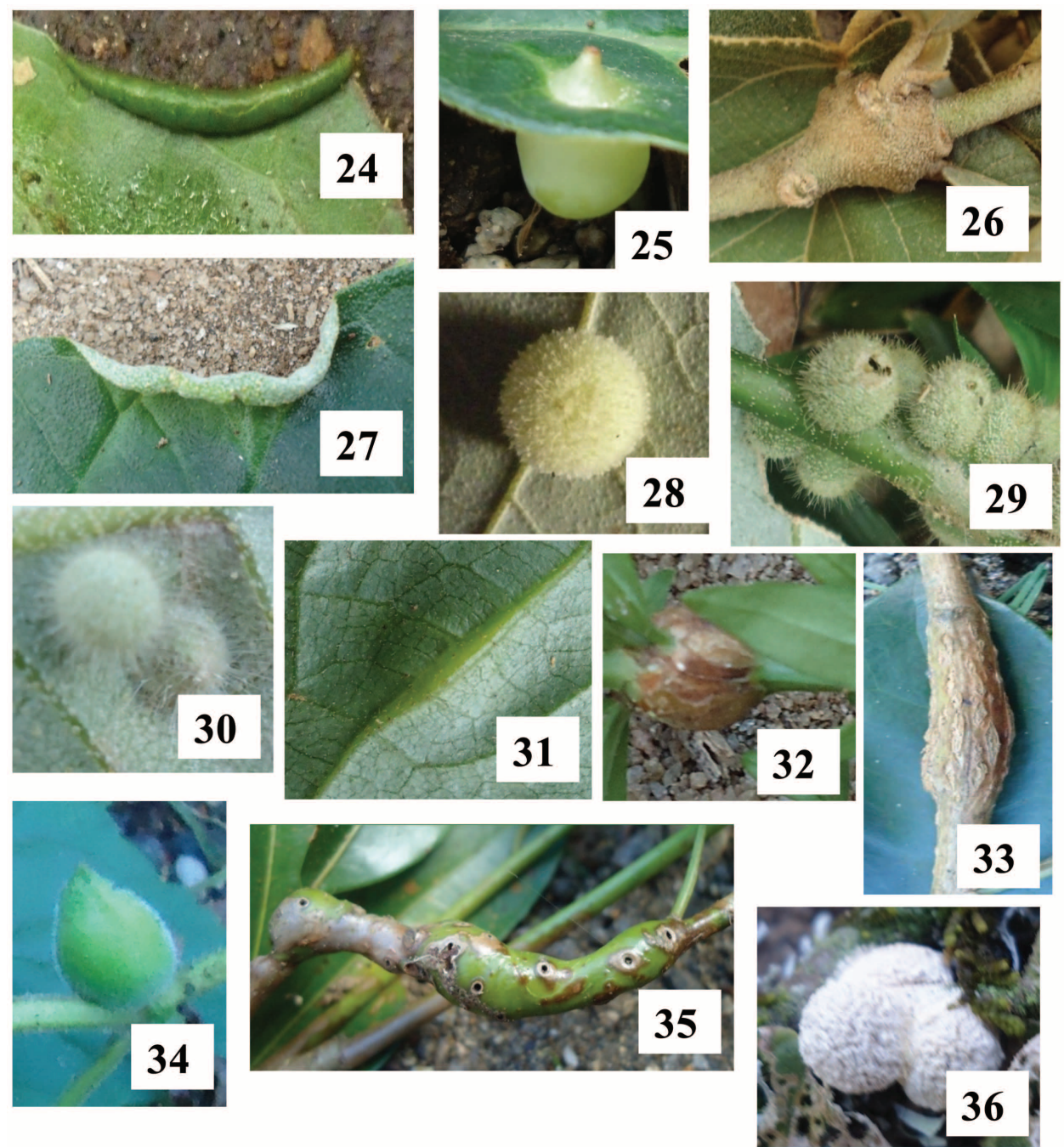

Figures 24-36. Insect galls of Itamonte (MG, Brazil):24. Marginal leaf roll on Protium heptaphyllum (Burseraceae); 25. Leaf gall on Centropogon sp. (Campanulaceae); 26. Stem gall on Croton sp.1 (Euphorbiaceae); 27. Marginal leaf roll on Croton sp.2 (Euphorbiaceae); 28. Leaf gall on Croton sp.2 (Euphorbiaceae); 29. Bud gall on Croton sp.2 (Euphorbiaceae); 30. Leaf gall on Croton sp.2 (Euphorbiaceae); 31. Vein gall on Inga sp. (Fabaceae); 32. Stem gall on Stylosanthes graciles (Fabaceae); 33. Stem gall on Humiriantheca sp. (Iracinaceae); 34. Bud gall on Hyptis sp. (Lammiaceae); 35. Stem gall on Licania sp. (Lauraceae); 36. Leaf gall on Nectandra sp. (Lauraceae).Figuras 24-36. Galhas de insetos de Itamonte (MG, Brasil): 24. Enrolamento da margem foliar em Protium heptaphyllum (Burseraceae); 25. Galha foliar em Centropogon sp. (Campanulaceae); 26. Galha caulinar em Croton sp.1 (Euphorbiaceae); 27. Enrolamento da margem foliar em Croton sp.2 (Euphorbiaceae); 28. Galha foliar em Croton sp.2 (Euphorbiaceae); 29. Galha da gema em Croton sp.2 (Euphorbiaceae); 30. Galha foliar em Croton sp.2 (Euphorbiaceae); 31 . Galha na nervura em Inga sp. (Fabaceae); 32. Galha caulinar em Stylosanthes graciles (Fabaceae); 33. Galha caulinar em Humiriantheca sp. (Iracinaceae); 34. Galha da gema em Hyptis sp. (Lammiaceae); 35. Galha caulinar em Licania sp. (Lauraceae); 36. Galha foliar em Nectandra sp. (Lauraceae). 
Croton sp. 2

Marginal leaf roll, green, glabrous, one-chambered (Figure 27). Galler: not determined. Locality: Cachoeira do Escorrega. Occurrence: December, June.

Leaf gall, globose, yellow, hairy, one-chambered (Figure 28). Galler: Cecidomyiidae (Diptera). Locality: Cachoeira do Escorrega. Occurrence: June.

Bud gall, globoid, yellow, hairy, gregarious, one-chambered (Figure 29). Galler: Cecidomyiidae (Diptera). Locality: Cachoeira do Escorrega. Occurrence: December

Leaf, petiole or stem gall, globose, yellow, hairy, onechambered (Figure 30) Galler: Cecidomyiidae (Diptera). Localities: Sitio Moana, Cachoeira do Escorrega. Occurrence: September, December, June.

Previous records: several galls on Croton spp. were recorded in Ceará, Pernambuco, Minas Gerais, and Rio de Janeiro (Rübsaamen 1905, Tavares 1922, 1925 Urso-Guimarães et al. 2003, Maia \& Fernandes 2004, Carneiro et al. 2009, Santos et al. 2011, Malves \& Frieiro-Costa 2012).

\section{Fabaceae}

Inga sp.

Vein swelling, fusiform, green, glabrous one-chambered (Figure 31). Galler: Neolasioptera sp. (Cecidomyiidae, Diptera). Locality: Cachoeira do Escorrega. Occurrence: December. Previous records: several galls on Inga sp. were recorded in Amazonas, Pará, Goiás, Mato Grosso do Sul, Minas Gerais, Rio de Janeiro, São Paulo, and Santa Catarina (Rübsaamen 1907, Tavares 1920b, Fernandes et al. 1988, Maia 2001, Julião et al. 2002, Urso-Guimarães et al. 2003, Maia \& Fernandes 2004, Oliveira \& Maia 2005, Maia et al. 2008, Maia \& Oliveira 2010, Santos et al. 2010, Maia 2011).

Stylosanthes graciles Kunth

Stem swelling, globose, green, glabrous, one-chambered (Figure 32). Galler: Cecidomyiidae (Diptera). Locality: Cachoeira do Escorrega. Occurrence: March.

Previous records: a stem gall on Stylosanthes sp. was recorded in Minas Gerais by Coelho et al. (2009).

\section{Iracinaceae}

Humiriantheca sp.

Stem swelling, fusiform, green, glabrous, one-chambered (Figure 33). Galler: Cecidomyiidae (Diptera). Locality: Brejo da Lapa. Occurrence: June.

\section{Lammiaceae}

Hyptis sp.

Bud gall, conical, green or yellowish, hairy, one-chambered (Figure 34). Galler: Cecidomyiidae (Diptera). Locality: Brejo da Lapa. Occurrence: December, March, June.

Previous records: galls on Hyptis spp. were recorded in Pernambuco, Minas Gerais, and São Paulo (Maia \& Fernandes 2004, Maia et al. 2008, Carneiro et al. 2009, Coelho et al. 2009, Santos et al. 2011).

\section{Lauraceae}

\section{Licania sp.}

Stem swelling, fusiform, green, glabrous, one-chambered (Figure 35). Galler: Cecidomyiidae (Diptera). Locality: Brejo da Lapa. Occurrence: June.

Previous records: galls on Licania spp. were recorded in
Pará, Goiás, Minas Gerais, and São Paulo (Maia \& Fernandes 2004, Maia et al. 2008, Santos et al. 2010, Maia 2011).

Nectandra sp.

Leaf gall, brown, globose, glabrous, one-chambered (Figure 36). Galler: Hemiptera. Locality: Brejo da Lapa. Occurrence: March, June.

Previous records: several galls were recorded on Nectandra spp. in Goiás, São Paulo, Santa Catarina, and Rio Grande do Sul (Rübsaamen 1908, Tavares 1909, 1921, Maia et al. 2008, Santos et al. 2010).

Ocotea cernua (Nees) Mez

Aerial root swelling, globose, brown, glabrous, onechambered (Figure 37). Galler: not determined. Locality: Sitio Moana. Occurrence: September

Previous records: Rübsaamen (1908), Monteiro et al. (1994), Maia (2001), Julião et al. (2002), Monteiro et al. (2004), Maia \& Fernandes (2004), Maia et al. (2008), and Carneiro et al. (2009) recorded galls on Ocotea spp. in Mato Grosso do Sul, Minas Gerais and Rio de Janeiro.

\section{Loranthaceae}

Phthirusa sp.

Leaf gall, globose, glabrous, green or red, one-chambered (Figure 38). Galler: Cecidomyiidae (Diptera) (1 larva on 11.XII.2011). Locality: Brejo da Lapa. Occurrence: September, December.

Leaf gall, circular, yellow, glabrous, one-chambered (Figure 39). Galler: not determined. Locality: Brejo da Lapa. Occurrence: September.

Aerial root swelling, fusiform, brown, glabrous, onechambered (Figure 40). Galler: Cecidomyiidae (Diptera). Locality: Brejo da Lapa. Occurrence: September.

Stem swelling, fusiform, glabrous, brown, one-chambered. Galler: Lepidoptera. Locality: Brejo da Lapa. Occurrence: September, December.

\section{Lythraceae}

Cuphea sp.

Stem swelling, fusiform, brown, glabrous, one-chambered (Figure 41). Galler: Cecidomyiidae (Diptera). Locality: Sítio Moana. Occurrence: September.

Previous record: Gagné (1994) and Carneiro et al. (2009) recorded galls on Cuphea spp. in Minas Gerais.

\section{Melastomataceae}

Aciotis cf. indecora (Bonpl) Triana

Vein swelling, fusiform, woody, brown, glabrous, onechambered (Figure 42). Galler: not determined. Locality: Cachoeira do Escorrega. Occurrence: June.

Stem swelling, brown, fusiform, glabrous, one-chambered (Figure 43). Galler: not determined. Locality: Cachoeira do Escorrega. Occurrence: June.

Bud gall, globose, green, micropubescent, one-chambered (Figure 44). Galler: not determined. Locality: Cachoeira do Escorrega. Occurrence: June.

Bud gall, globose, green, macropubescent (reddish trichomes), one-chambered (Figure 45). Galler: not determined. Locality: Cachoeira do Escorrega. Occurrence: June.

Clidemia capitellata (Bonpl) D.Don

Leaf gall, globose, green, hairy, one-chambered (Figure 46). Galler: Cecidomyiidae (Diptera). Locality: Cachoeira do Escorrega. Occurrence: June. 

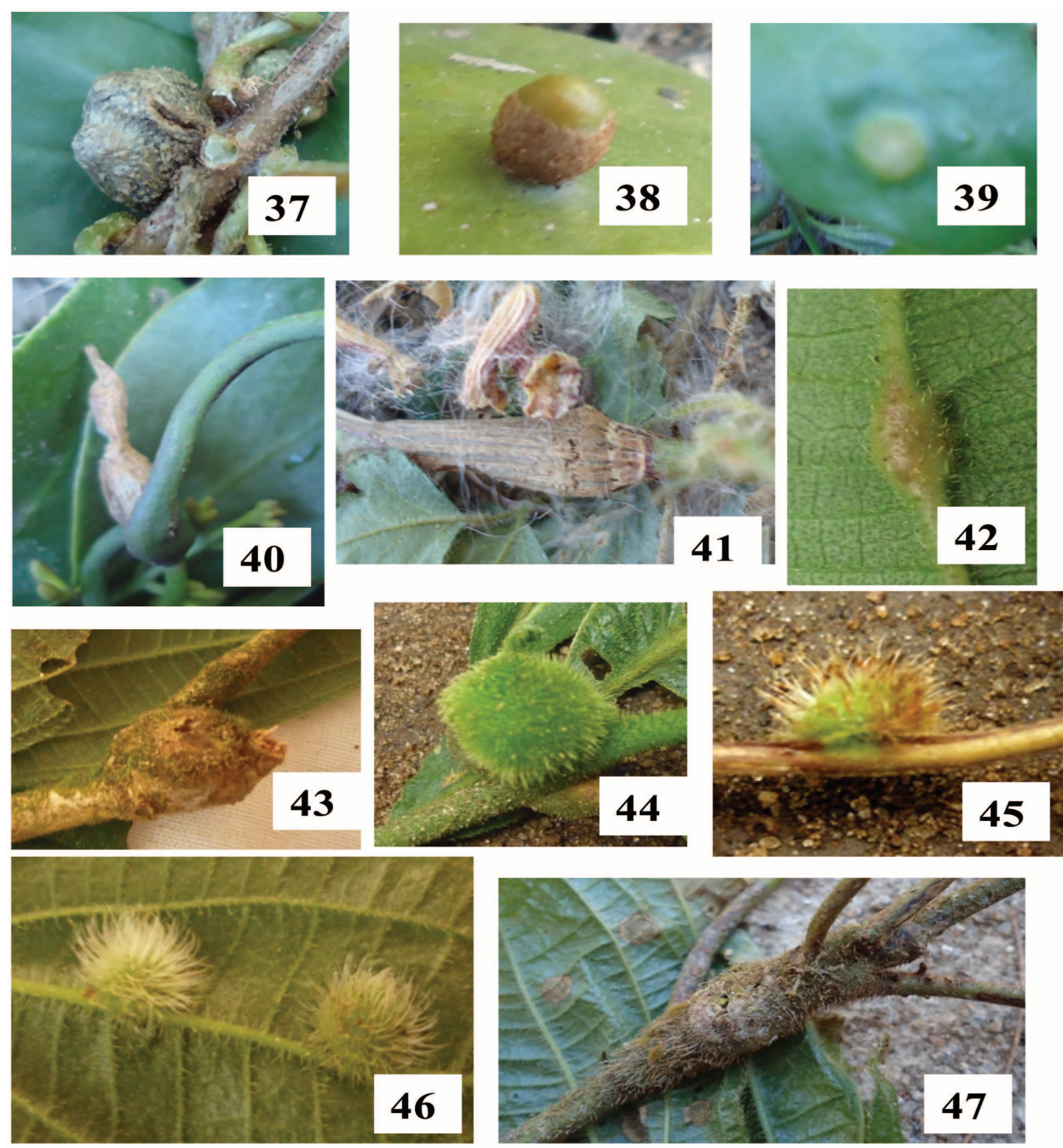

Figures 37-47. Insect galls of Itamonte (MG, Brazil): 37. Aerial root gall on Ocotea cernua (Lauraceae); 38. Leaf gall on Phthirusa sp. (Loranthaceae); 39. Leaf gall on Phthirusa sp. (Loranthaceae); 40. Aerial root gall on Phthirusa sp. (Loranthaceae); 40. Stem gall on Phthirusa sp. (Loranthaceae); 41. Stem swelling on Cuphea sp. (Lythraceae); 42. Vein gall on Aciotis cf. indecora (Melastomataceae); 43. Stem gall on Aciotis cf. indecora (Melastomataceae); 44. Bud gall on Aciotis cf. indecora (Melastomataceae); 45. Bud gall on Aciotis cf. indecora (Melastomataceae); 46. Leaf gall on Clidemia capitellata (Melastomataceae); 47. Stem swelling on Clidemia sp.1 (Melastomataceae). Figuras 37-47. Galhas de insetos de Itamonte (MG, Brasil): 37. Galha em raízes aéreas em Ocotea cernua (Lauraceae); 38. Galha foliar em Phthirusa sp. (Loranthaceae); 39. Galha foliar em Phthirusa sp. (Loranthaceae); 40. Galha em raízes aéreas em Phthirusa sp. (Loranthaceae); 40. Galha caulinar em Phthirusa sp. (Loranthaceae); 41. Galha caulinar em Cuphea sp. (Lythraceae); 42. Galha na nervura em Aciotis cf. indecora (Melastomataceae); 43. Galha caulinar em Aciotis cf. indecora (Melastomataceae); 44. Galha da gema em Aciotis cf. indecora (Melastomataceae); 45. Galha da gema em Aciotis cf. indecora (Melastomataceae); 46. Galha foliar em Clidemia capitellata (Melastomataceae); 47. Galha caulinar em Clidemia sp.1 (Melastomataceae).

\section{Clidemia sp. 1}

Stem swelling, fusiform, brown, hairy, one-chambered (Figure 47). Galler: not determined. Locality: Sítio Moana. Occurrence: September.

Lateral bud or leaf gall, globose, yellow, hairy (reddish trichomes), one-chambered. Galler: Cecidomyiidae (Diptera). Locality: Cachoeira do Escorrega. Occurrence: March, June.

Clidemia sp. 2

Stem swelling, fusiform, gregarious, glabrous, brown, multichambered (Figure 48). Galler: Cecidomyiidae (Diptera). Associated fauna: parasitoid - Hymenoptera. Locality: Brejo da Lapa. Occurrence: December, March.

Leaf gall, globose, green, hairy (whitish trichomes), onechambered (Figure 49). Galler: Cecidomyiidae (Diptera). Locality: Brejo da Lapa. Occurrence: March.

Previous records: Tavares (1917a) and Maia et al. (2008) recorded galls on Clidemia spp.

Miconia cf. cinnamomifolia (DC.) Naudin

Leaf gall, globose, hairy, one-chambered (Figure 50). Galler: Cecidomyiidae (Diptera). Locality: Cachoeira do Escorrega. Occurrence: December, March.

Miconia cf. latecrenata (DC.) Naudin

Bud gall, pedunculate, globose, rough, glabrous, onechambered (Figure 51). Galler: not determined. Locality: Sítio Moana. Occurrence: September.

Leaf gall, circular, yellowish, glabrous, one-chambered (Figure 52). Galler: Cecidomyiidae (Diptera). Locality: Cachoeira do Escorrega. Occurrence: December, March.

Miconia sp.1

Midvein or petiole swelling, fusiform, green, glabrous, onechambered (Figures 53 and 54). Galler: not determined. Locality: Brejo da Lapa. Occurrence: March. 

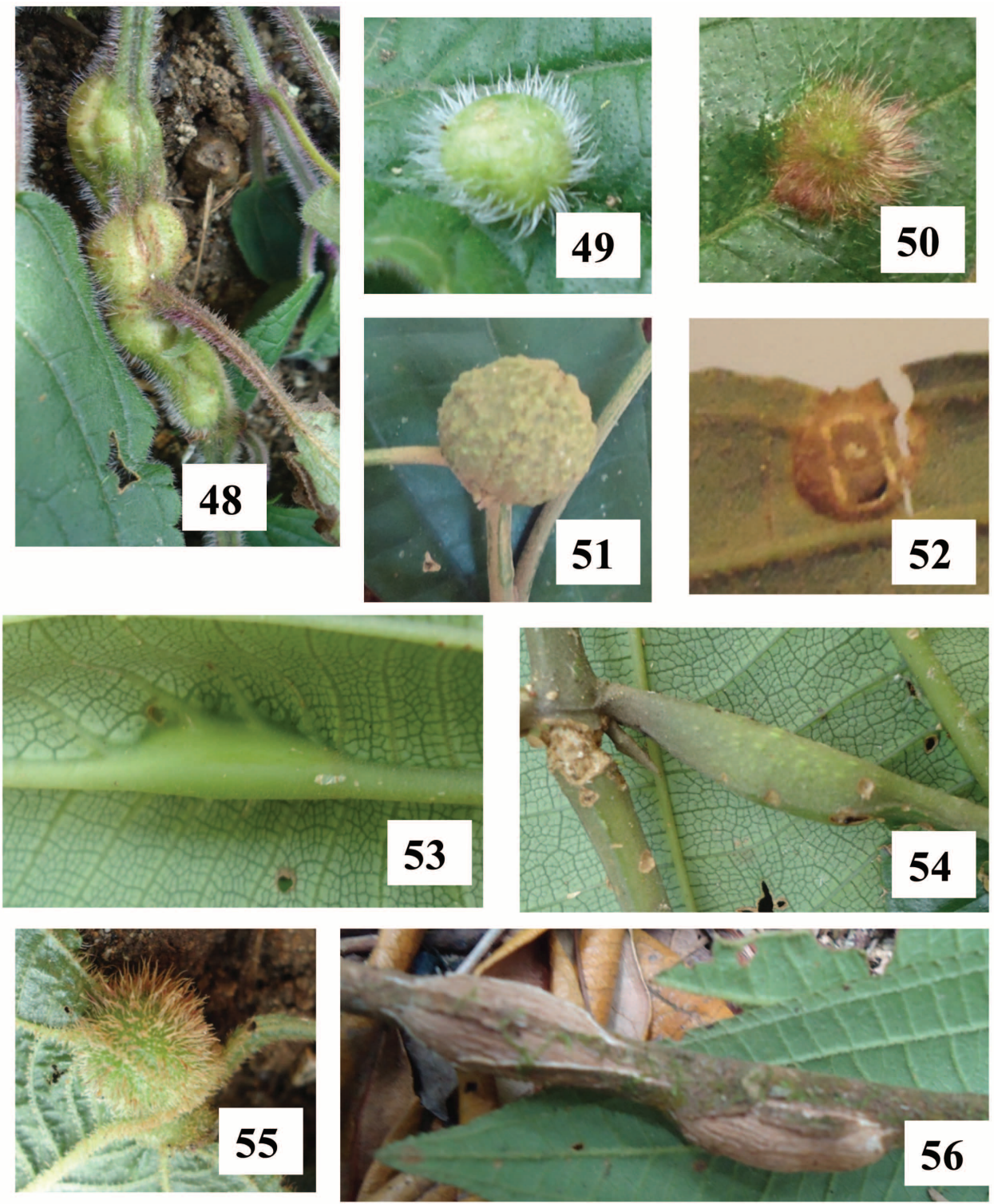

Figures 48-56. Insect galls of Itamonte (MG, Brazil): 48. Stem gall on Clidemia sp. 2 (Melastomataceae); 49. Leaf gall on Clidemia sp. 2 (Melastomataceae); 50. Leaf gall on Miconia cf. cinnamomifolia (Melastomataceae); 51. Bud gall on Miconia cf. latecrenata (Melastomataceae); 52. Leaf gall on Miconia cf. latecrenata (Melastomataceae); 53. Midvein gall on Miconia sp.1 (Melastomataceae); 54. Petiole gall on Miconia sp.1 (Melastomataceae); 55. Leaf gall on Miconia sp.2 (Melastomataceae); 56. Stem gall on Miconia sp.3 (Melastomataceae).Figuras 48-56. Galhas de insetos de Itamonte (MG, Brasil): 48. Galha caulinar em Clidemia sp. 2 (Melastomataceae); 49. Galha foliar em Clidemia sp. 2 (Melastomataceae); 50. Galha foliar em Miconia cf. cinnamomifolia (Melastomataceae); 51. Galha da gema em Miconia cf. latecrenata (Melastomataceae); 52. Galha foliar em Miconia cf. latecrenata (Melastomataceae); 53. Galha da nervura central em Miconia sp.1 (Melastomataceae); 54. Galha peciolar em Miconia sp.1 (Melastomataceae); 55. Galha foliar em Miconia sp.2 (Melastomataceae); 56. Galha caulinar em Miconia sp.3 (Melastomataceae).

\section{Miconia sp. 2}

Leaf or bud gall, globose, green with reddish trichomes, one-chambered (Figure 55). Galler: Cecidomyiidae (Diptera). Locality: Brejo da Lapa. Occurrence: September, December, March.

\section{Miconia sp.3}

Stem swelling, fusiform, brown, glabrous, one-chambered (Figure 56). Galler: not determined. Locality: Cachoeira do Escorrega. Occurrence: December.

Bud gall, globose, reddish, hairy, one-chambered. Galler: Lepidoptera. Locality: Cachoeira do Escorrega. Occurrence: December.

\section{Miconia sp. 4}

Stem swelling, fusiform, brown, glabrous, one-chambered
(Figure 57). Galler: not determined. Locality: Cachoeira do Escorrega. Occurrence: December.

Leaf gall, globose, red, hairy, gregarious, one-chambered. Galler: Cecidomyiidae (Diptera). Locality: Cachoeira do Escorrega. Occurrence: December.

Miconia sp.5

Leaf gall, globose, brownish, glabrous, one-chambered (Figure 58). Galler: not determined. Locality: Cachoeira do Escorrega. Occurrence: December.

Miconia sp.6

Bud gall, globose, yellowish or reddish, hairy, onechambered (Figure 59). Galler: Cecidomyiidae (Diptera). Locality: Cachoeira do Escorrega. Occurrence: December, March. 

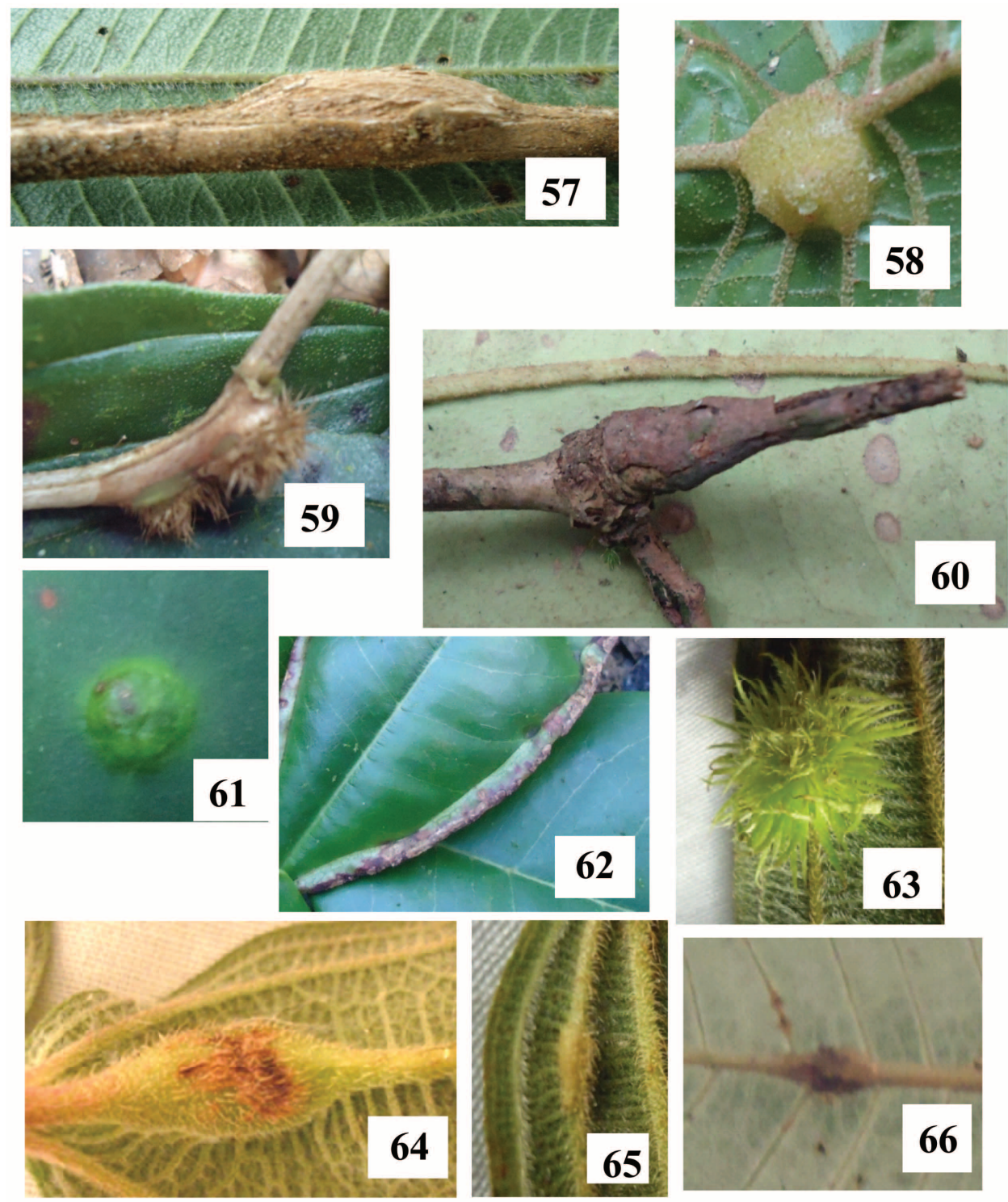

Figures 57-66. Insect galls of Itamonte (MG, Brazil): 57. Stem gall on Miconia sp.4 (Melastomataceae); 58. Leaf gall on Miconia sp.5 (Melastomataceae); 59. Bud gall on Miconia sp.6 (Melastomataceae); 60. Stem gall on Mouriri sp. (Melastomataceae); 61. Leaf gall on Mouriri sp. (Melastomataceae); 62. Marginal leaf roll on Mouriri sp. (Melastomataceae); 63. Leaf gall on Tibouchina cf. stenocarpa (Melastomataceae); 64. Vein gall on Tibouchina cf. stenocarpa (Melastomataceae); 65. Vein gall on Tibouchina cf. stenocarpa (Melastomataceae); 66. Midvein gall on Melastomataceae not determined.Figuras 57-66. Galhas de insetos de Itamonte (MG, Brasil): 57. Galha caulinar em Miconia sp.4 (Melastomataceae); 58. Galha foliar em Miconia sp.5 (Melastomataceae); 59. Galha da gema em Miconia sp.6 (Melastomataceae); 60. Galha caulinar em Mouriri sp. (Melastomataceae); 61. Galha foliar em Mouriri sp. (Melastomataceae); 62. Enrolamento da margem foliar em foliar Mouriri sp. (Melastomataceae); 63. Galha foliar em Tibouchina cf. stenocarpa (Melastomataceae); 64. Galha da nervura em Tibouchina cf. stenocarpa (Melastomataceae); 65. Galha da nervura em Tibouchina cf. stenocarpa (Melastomataceae); 66. Galha da nervura central em Melastomataceae não determinada.

Previous records: several different kinds of gall were recorded in Amazonas, Pará, Minas Gerais, São Paulo and Rio de Janeiro (Rübsaamen 1907, Tavares 1917a, Maia 2001a, Monteiro et al. 2004, Maia \& Fernandes 2004,Urso-Guimarães \& Scareli-Santos 2006, Maia et al. 2008; Carneiro et al. 2009, Maia 2011, Malves \& Frieiro-Costa 2012).

Mouriri sp.

Stem swelling, fusiform, brown, glabrous, one-chambered (Figure 60). Galler: not determined. Locality: Cachoeira do Escorrega. Occurrence: December.

Leaf gall, circular, yellow, glabrous, one-chambered (Figure 61). Galler: Cecidomyiidae (Diptera). Locality: Cachoeira do
Escorrega. Occurrence: December, March, June.

Marginal leaf roll, reddish, glabrous, one-chambered (Figure 62). Galler: Cecidomyiidae (Diptera). Locality: Brejo da Lapa. Occurrence: June.

Previous records: Rübsaamen 1908 described a flower gall, probably induced by Asphondylia sp. (Cecidomyiidae) on Mouriri ulei from Amazonas state (Brazil).

Tibouchina cf. stenocarpa (DC.) Cogn.

Leaf gall, globose, green, hairy, one-chambered (Figure 63). Galler: Lepidoptera. Locality: Cachoeira do Escorrega. Occurrence: March, June.

Vein swelling, fusiform, conspicuous, green, glabrous, one- 
chambered (Figure 64). Galler: Lepidoptera. Locality: Cachoeira do Escorrega. Occurrence: March, June.

Vein swelling, linear, barely evident, green, glabrous, onechambered (Figure 65). Galler: not determined. Locality: Cachoeira do Escorrega. Occurrence: June.

Stem swelling, fusiform, brown, glabrous, one-chambered. Galler. Not determined. Locality: Cachoeira do Escorrega. Occurrence: March.

Previous records: galls on other Tibouchina spp. were recorded in Minas Gerais, São Paulo, and Rio de Janeiro (Rübsaamen, 1908; Tavares, 1917a, Maia \& Fernandes, 2004; Maia et al. 2008).
Melastomataceae not determined

Midvein swelling, fusiform, brown, glabrous, one-chambered (Figure 66). Galler: not determined. Locality: Brejo da Lapa. Occurrence: December.

Leaf gall, globose, green, glabrous, one-chambered (Figure 67). Galler: not determined. Locality: Cachoeira do Escorrega. Occurrence: June.

\section{Myrtaceae}

Eugenia uniflora L.

Leaf gall, circular, green or yellow, glabrous, one-chambered (Figure 68). Galler: Neolasioptera eugeniae Maia, 1993
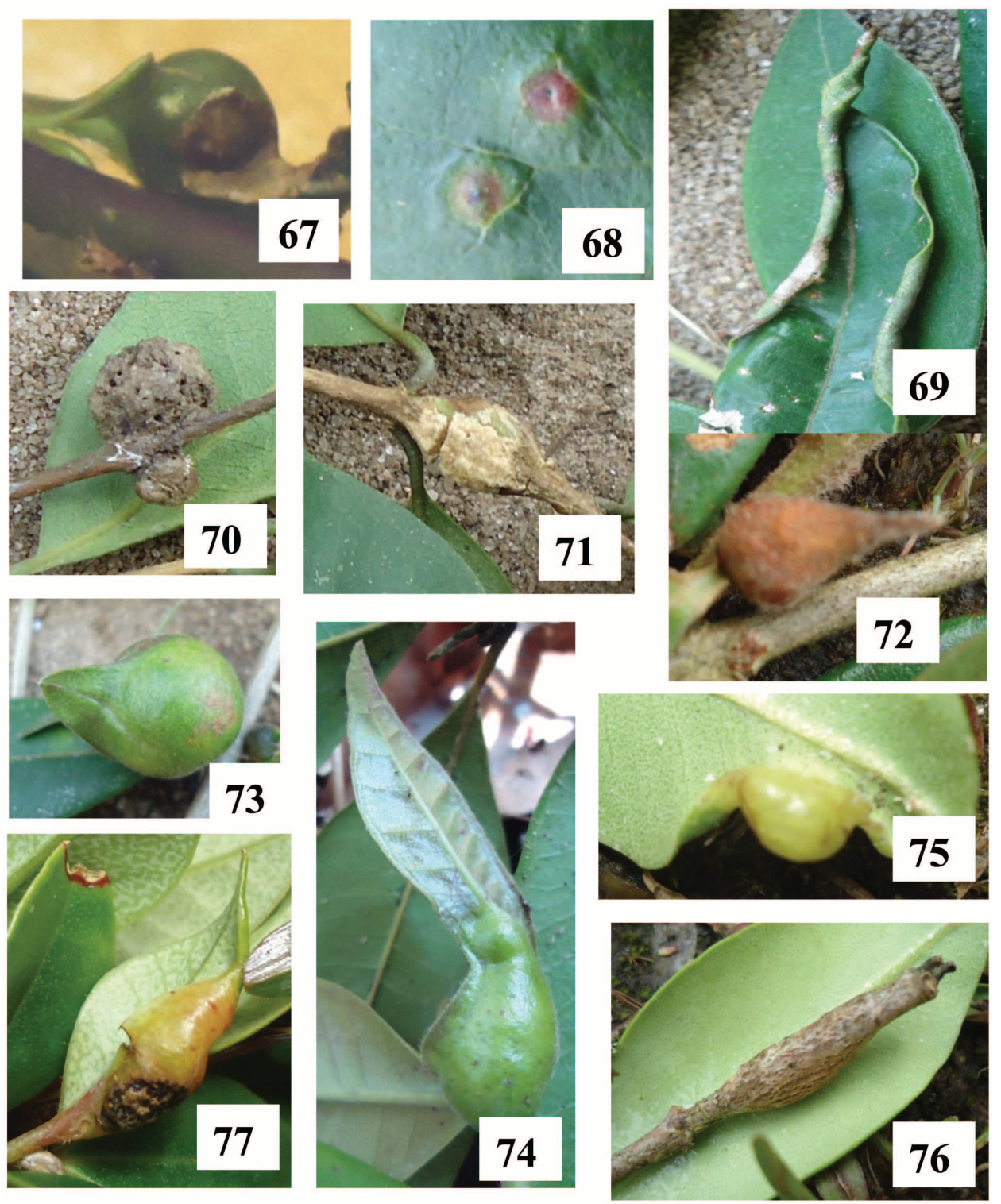

Figures 67-77. Insect galls of Itamonte (MG, Brazil): 67. Leaf gall on Melastomataceae not determined; 68. Leaf gall on Eugenia uniflora (Myrtaceae); 69. Marginal leaf gall on Marlierea sp. (Myrtaceae); 70. Lateral bud gall on Marlierea sp. (Myrtaceae); 71. Stem gall on Marlierea sp. (Myrtaceae); 72. Apical bud gall on Marlierea sp. (Myrtaceae); 73. Lateral bud gall on Marlierea sp. (Myrtaceae); 74. Leaf gall on Myrcia fallax (Myrtaceae); 75. Marginal leaf gall on Myrcia sp. (Myrtaceae); 76. Apical bud gall on Myrcia sp. (Myrtaceae); 77. Marginal leaf gall on Myrciaria sp. (Myrtaceae).Figuras 67-77. Galhas de insetos de Itamonte (MG, Brasil): 67.Galha foliar em Melastomataceae não determinada; 68. Galha foliar em Eugenia uniflora (Myrtaceae); 69. Enrolamento da margem foliar em Marlierea sp. (Myrtaceae); 70. Galha da gema lateral em Marlierea sp. (Myrtaceae); 71. Galha caulinar em Marlierea sp. (Myrtaceae); 72. Galha da gema apical em Marlierea sp. (Myrtaceae); 73. Galha da gema lateral em Marlierea sp. (Myrtaceae); 74. Galha foliar em Myrcia fallax (Myrtaceae); 75. Enrolamento da margem foliar em Myrcia sp. (Myrtaceae); 76. Galha da gema apical em Myrcia sp. (Myrtaceae); 77. Enrolamento da margem foliar em Myrciaria sp. (Myrtaceae). 
(Cecidomyiidae, Diptera). Cachoeira do Escorrega. Occurrence: December.

Previous records: the same gall was recorded in the several localities of the State of Rio de Janeiro (Monteiro et al. 1994, Maia 2001a, Monteiro et al. 2004, Oliveira \& Maia 2005, Maia \& Oliveira 2010).

\section{Marlierea $\mathrm{sp}$.}

Marginal leaf gall, green, glabrous, one-chambered (Figure 69). Galler: Cecidomyiidae (Diptera). Locality: Cachoeira do Escorrega. Occurrence: December

Lateral bud gall, globose, brown, glabrous, multichambered (Figure 70). Galler: not determined. Locality: Brejo da Lapa, Cachoeira do Escorrega. Occurrence: December, March, June.

Stem swelling, fusiform, brown, glabrous, one-chambered (Figure 71). Galler: Cecidomyiidae (Diptera). Locality: Cachoeira do Escorrega. Occurrence: December, March, June.

Apical bud gall, conical, brownish or reddish, glabrous, one-chambered (Figure 72). Galler: not determined. Locality: Cachoeira do Escorrega. Occurrence: December

Lateral and apical bud gall, conical, green, glabrous, onechambered (Figure 73). Galler: not determined. Associated fauna: successors - ants (adults) (Hymenoptera). Locality: Cachoeira do Escorrega. Occurrence: December, March.

Myrcia fallax (Rich) DC.

Leaf gall, globose, green, glabrous, one-chambered (Figure 74). Galler: Cecidomyiidae (Diptera). Locality: Sítio Moana. Occurrence: September. Previous records: Maia et al. (2008) recorded seven different galls on this host plant in São Paulo.

Myrcia pubipetala Miq.

Marginal leaf roll, green, glabrous, one-chambered. Galler: Cecidomyiidae (Diptera). Locality: Cachoeira do Escorrega. Occurrence: June.

\section{Myrcia sp.}

Marginal leaf gall, green, glabrous, one-chambered (Figure 75). Galler: Hemiptera. Locality: Brejo da Lapa. Occurrence: March.

Apical bud gall, fusiform, brown, glabrous, one-chambered (Figure 76). Galler: Lepidoptera. Locality: Brejo da Lapa. Occurrence: March.

Previous records: galls on Myrcia spp. were recorded in Goiás, Minas Gerais, Rio de Janeiro, and São Paulo (Maia 2001a, Maia et al. 2002, Maia \& Fernandes 2004, Monteiro et al. 2004, Araujo et al. 2007, Maia et al. 2008, Carneiro et al. 2009, Coelho et al. 2009, Santos et al. 2010, Malves \& FrieiroCosta 2012).

Myrciaria sp.

Marginal leaf gall, green, glabrous, one-chambered (Figure 77). Galler: Hemiptera. Locality: Brejo da Lapa. Occurrence: March. Previous records: Galls on Myrciaria spp. were recorded in Minas Gerais, Espírito Santo, and Rio de Janeiro (Gagné 1994, Monteiro et al. 1994, Maia 2001a, Maia \& Fernandes 2004, Monteiro et al. 2004, Bregonci et al. 2010, Maia \& Oliveira 2010).

\section{Nyctaginaceae}

\section{Neea sp.}

Leaf gall, circular, green, glabrous, one-chambered (Figure 78). Galler: Bruggmannia sp. (Cecidomyiidae, Diptera). Locality: Cachoeira do Escorrega. Occurrence: December, March. Previous records: galls on Neea sp. were recorded in Amazonas, Mato Grosso do Sul, Rio de Janeiro, and Santa Catarina (Rübsaamen 1908, Julião et al. 2002).

\section{Piperaceae}

Piper sp.

Stem swelling, globose or fusiform, green, glabrous, multichambered (Figure 79). Galler:.not determined. Locality: Sítio Moana. Occurrence: September.

Leaf gall, globose, green, glabrous, one-chambered (Figure 80). Galler: Cecidomyiidae (Diptera) Locality: Sítio Moana. Occurrence: September.

Bud gall, globose, brown, glabrous, one-chambered (Figure 81). Galler: not determined. Associated fauna: parasitoid Hymenoptera. Locality: Cachoeira do Escorrega. Occurrence: December.

Previous records: different galls on Piper spp. were recorded in Goiás, Minas Gerais, São Paulo, and Rio de Janeiro (Rübsaamen 1908, Tavares 1909, 1925, Maia 2001, Maia \& Fernandes 2004, Oliveira \& Maia 2005, Araujo et al. 2007, Maia et al. 2008, Santos et al. 2010).

\section{Proteaceae}

Roupala montana var. brasiliensis (Klotzsch) K. S. Edwards Leaf fold, green, glabrous, one-chambered (Figure 82). Galler: not determined. Locality: Brejo da Lapa. Occurrence: June.

Roupala montana var. dentata (R. BR.) Sleumer

Leaf gall, conical, glabrous, green, one-chambered (Figure 83). Galler: not determined. Locality: Cachoeira do Escorrega. Occurrence: June.

Previous records: a hemipterous gall on Roupala montana was recorded in Goiás by Araujo et al. (2007).

\section{Rubiaceae}

Pagamea sp.

Stem swelling, fusiform, whittish, glabrous, one-chambered (Figure 84). Galler: Cecidomyiidae (Diptera). Locality: Cachoeira do Escorrega. Occurrence: December, March, June.

Palicourea sp.

Leaf gall, conical, green, glabrous, one-chambered (Figure 85). Galler: Cecidomyiidae (Diptera). Locality: Cachoeira do Escorrega. Occurrence: December, March, June. Previous record: Carneiro et al. (2009) recorded a globose leaf gall on Palicourea rigida Kunth in Minas Gerais.

Psychotria sp.

Leaf gall, green, globose, glabrous, succulent, one-chambered (Figure 86). Galler: not determined. Localities: Brejo da Lapa, Cachoeira do Escorrega. Occurrence: December, March.

Previous records: several galls were recorded in Mato Grosso do Sul, Minas Gerais, Rio de Janeiro, São Paulo and Rio Grande do Sul (Rübsaamen 1908, Julião et al. 2002, Carneiro et al. 2009, Tavares 1909, 1922, Maia 2008).

Rubiaceae not determined

Leaf gall, globose, green, glabrous, one-chambered (Figure 87). Galler: Cecidomyiidae (Diptera). Locality: Cachoeira do Escorrega. Occurrence: December.

\section{Salicaceae}

\section{Homalium sp.}

Marginal leaf roll, green, glabrous, one-chambered (Figure 88). Galler: Hemiptera. Locality: Cachoeira do Escorrega. Occurrence: December.

Young leaf roll, green, glabrous, one-chambered (Figure 89). Galler: Hemiptera. Locality: Brejo da Lapa. Occurrence: March, June. 

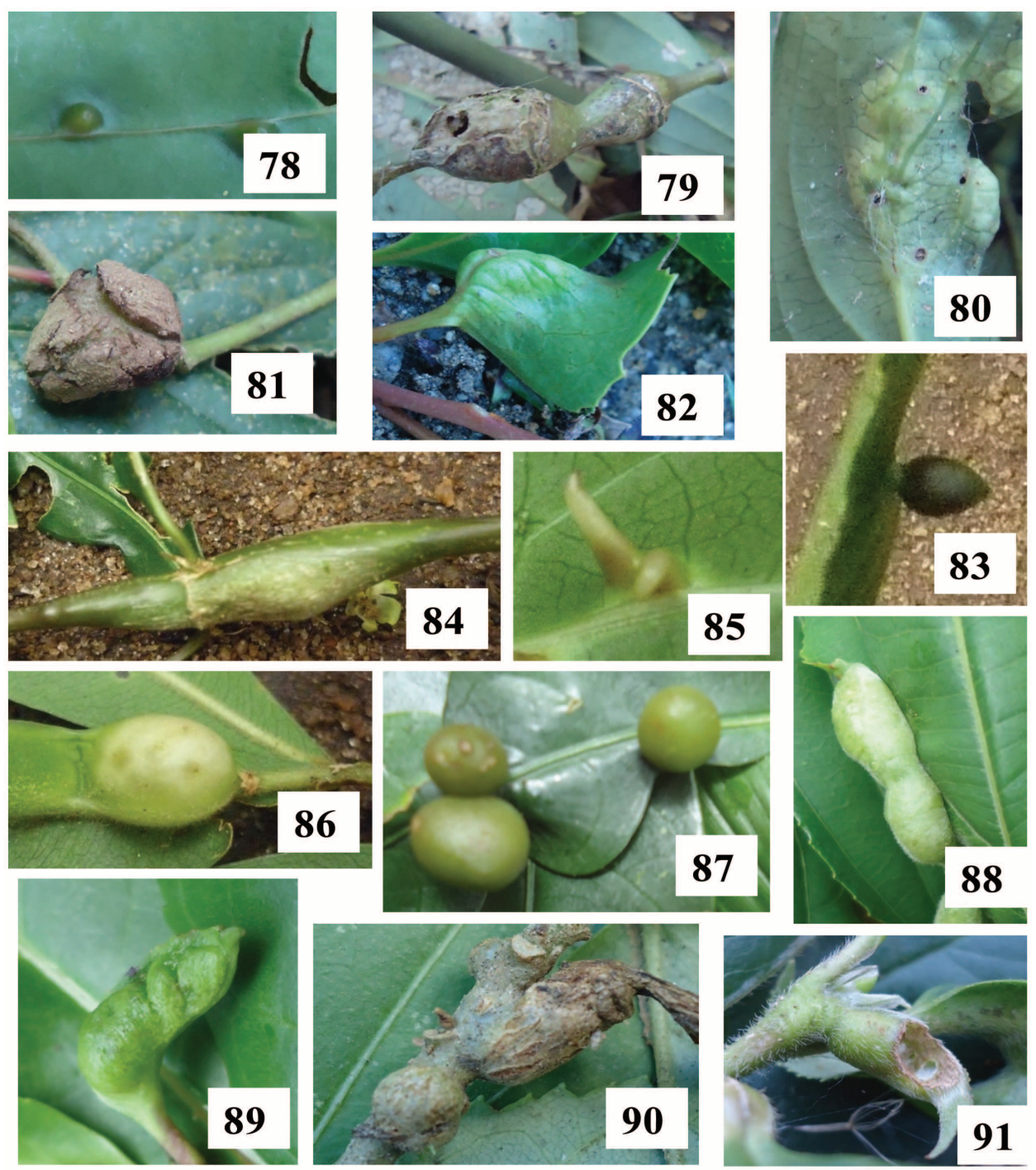

Figures 78-91. Insect galls of Itamonte (MG, Brazil): 78. Leaf gall on Neea sp. (Nyctaginaceae); 79. Stem gall on Piper sp. (Piperaceae); 80. Leaf gall on Piper sp. (Piperaceae); 81. Bud gall on Piper sp. (Piperaceae); 82. Leaf fold on Roupala montana var. brasiliensis (Proteaceae); 83. Leaf gall on Roupala montana var. dentata (Proteaceae); 84. Stem gall on Pagamea sp. (Rubiaceae); 85. Leaf gall on Palicourea sp. (Rubiaceae); 86. Leaf gall on Psychotria sp. (Rubiaceae); 87. Leaf gall on Rubiaceae not determined; 88. Marginal leaf roll on Homalium sp. (Salicaceae); 89. Young leaf roll on Homalium sp. (Salicaceae); 90. Stem gall on Xylosma sp. (Salicaceae); 91. Bud gall on Xylosma sp. (Salicaceae).Figuras 78-91. Galhas de insetos de Itamonte (MG, Brasil): 78. Galha foliar em Neea sp. (Nyctaginaceae); 79. Galha caulinar em Piper sp. (Piperaceae); 80. Galha foliar em Piper sp. (Piperaceae); 81. Galha da gema em Piper sp. (Piperaceae); 82. Dobramento da folha em Roupala montana var. brasiliensis (Proteaceae); 83. Galha foliar em Roupala montana var. dentata (Proteaceae); 84. Galha caulinar em Pagamea sp. (Rubiaceae); 85. Galha foliar em Palicourea sp. (Rubiaceae); 86. Galha foliar em Psychotria sp. (Rubiaceae); 87. Galha foliar em Rubiaceae não determinada; 88. Enrolamento da margem foliar em Homalium sp. (Salicaceae); 89. Enrolamento da folha jovem em Homalium sp. (Salicaceae); 90. Galha caulinar em Xylosma sp. (Salicaceae); 91. Galha da gema em Xylosma sp. (Salicaceae).

\section{Xylosma sp.}

Stem, midvein or petiole swelling, fusiform, green, glabrous, one-chambered (Figure 90). Galler: Cecidomyiidae (Diptera). Locality: Brejo da Lapa. Occurrence: June.

Bud gall, conical, green, glabrous, one-chambered (Figure 91). Galler: Hemiptera. Locality: Brejo da Lapa. Occurrence: June.

Previous record: Julião et al. (2002) recorded a leaf gall on Xylosma venosum.

\section{Sapindaceae}

Serjania sp.

Midvein swelling, fusiform, green, glabrous, one-chambered
(Figure 92). Galler: Cecidomyiidae (Diptera). Locality: Cachoeira do Escorrega. Occurrence: December.

Previous records: different galls were recorded on Serjania spp. in Amazonas, Goiás, Mato Grosso do Sul, Pernambuco, Minas Gerais, and São Paulo (Rübsaamen 1908, 1916a, Julião et al. 2002, Maia et al. 2008, Coelho et al. 2009, Santos et al. 2011).

\section{Solanaceae}

Solanum sp.1

Stem swelling fusiform, brown, glabrous, one-chambered (Figure 93). Galler: not determined. Locality: Brejo da Lapa. Occurrence: March. 

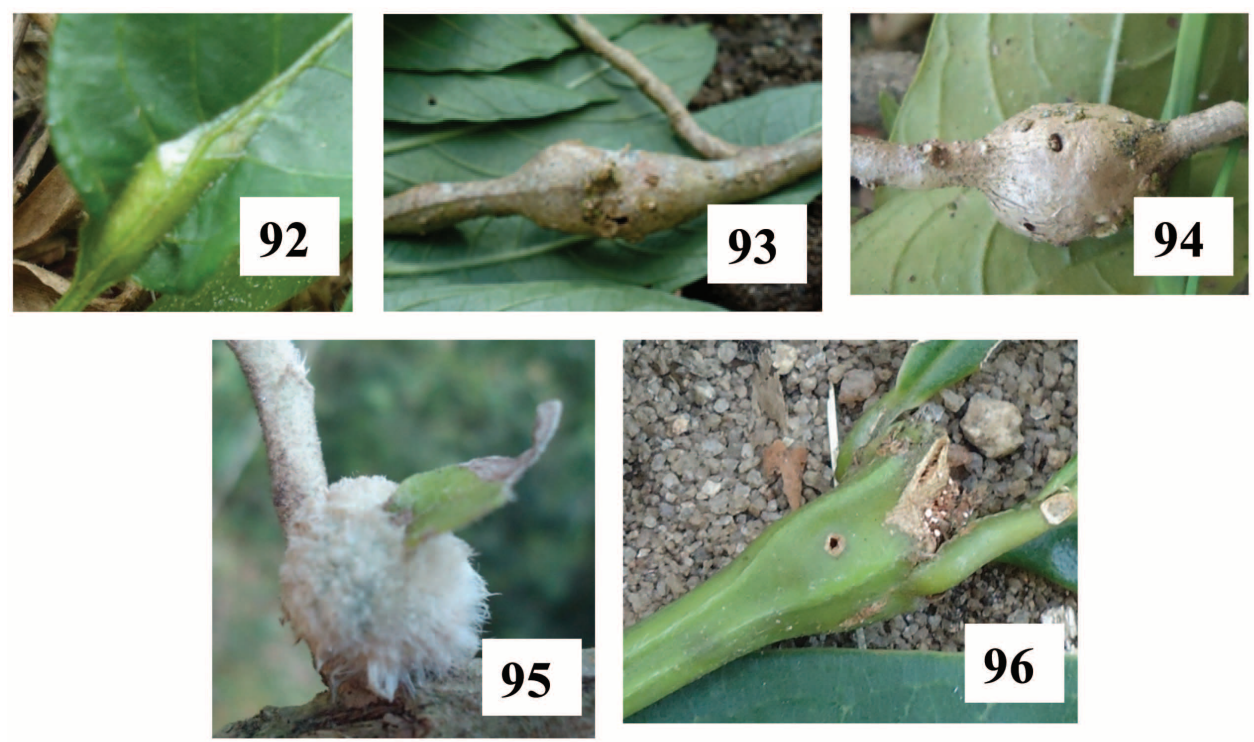

Figures 92-96. Insect galls of Itamonte (MG, Brazil): 92. Midvein gall on Serjania sp. (Sapindaceae); 93. Stem gall on Solanum sp.1 (Solanaceae); 94. Stem gall on Solanum sp.1 (Solanaceae); 95. Bud gall on Solanum sp.2 (Solanaceae); 96. Bud gall on Vochysia sp. (Vochysiaceae).Figuras 92-96. Galhas de insetos de Itamonte (MG, Brasil): 92. Galha da nervura central em Serjania sp. (Sapindaceae); 93. Galha caulinar em Solanum sp.1 (Solanaceae); 94. Galha caulinar em Solanum sp.1 (Solanaceae); 95. Galha da gema em Solanum sp.2 (Solanaceae); 96. Galha da gema em Vochysia sp. (Vochysiaceae).

Stem swelling, globose, brown, glabrous, one-chambered (Figure 94). Galler: Cecidomyiidae (Diptera). Locality: Brejo da Lapa. Occurrence: September.

Solanum sp. 2

Bud gall, globose, whittish, hairy, one-chambered (Figure 95). Galler: Cecidomyiidae (Diptera). Locality: Cachoeira do Escorrega. Occurrence: December.

Previous records: other galls on Solanum spp. were recorded in Pernambuco, Bahia, São Paulo and Rio de Janeiro by Rübsaamen (1908), Tavares (1918a), Monteiro et al. (1994), Maia (2001a), Monteiro et al. (2004), Oliveira \& Maia (2005), Maia et al. (2008), Santos et al. (2011), Malves \& Frieiro-Costa (2012).

\section{Vochysiaceae}

Vochysia sp.

Bud or stem gall, fusiform, green, glabrous, one-chambered (Figure 96). Galler: not determined. Locality: Cachoeira do Escorrega. Occurrence: March. Previous records: Carneiro et al. (2009) recorded galls on Vochysia spp. in Minas Gerais.

\section{Discussion}

Although several sampling methods and different collecting efforts have been employed in galls inventory in Brazil, Itamonte can be considered as an area of high richness of insect galls,overtaking several other areas in Brazilian states, such as Goiás (Parque Estadual da Serra dos Pireneus in Pirenópolis, and Campus Samambaia, Universidade Federal de Goiás, Goiânia) (Araújo et al. 2007, Santos et al. 2010), Minas Gerais (Serra do Cipó, Delfinópolis, Aimorés, and Jequitinhonha Valley) (Coelho et al. 2009, Urso-Guimarães et al. 2003, Fernandes \& Negreiros 2006, Fernandes et al. 1997), São Paulo (Pé-de-Gigante Cerrado Reserve in Santa Rita do Passa-Quatro, and Ecological Station of Jataí in Luiz Antônio) (Urso-Guimarães \& Scarelli-Santos 2006, Saito \& UrsoGuimarães 2012), Espírito Santo (Parque Estadual Paulo
César Vinha in Guarapari (Bregonci et al. 2010), and Rio de Janeiro (Reserva Biológica da Praia do Sul in Ilha Grande Angra dos Reis, Maricá, Carapebus, Grumari - Rio de Janeiro, and Arraial do Cabo) (Maia \& Oliveira 2010, Maia 2001, Oliveira \& Maia 2005, Monteiro et al. 1994).

The above cited areas comprise cerrado and restinga vegetation, and are considered as xeric environments with hygrotermical stress. One of the hypothesis on the adaptive nature of the insect galls (microenvironment hypothesis) predicts that xeric environments have higher gall diversity than mesic ones (Price et al. 1986). The surveyed areas of Itamonte are not xeric, and they are richer in gall morphotypes than those areas. Inventories in Amazonian forest (Julião 2007 and Oda 2006) showed an unexpected high diversity of galls, overtaking all other Brazilian biomes. So, the microenvironment hypothesis does not explain the pattern found in South America. The plant diversity hypothesis (Fernandes, 1992), which predicts a positive relation between plant diversity and gall diversity appears to be the most adequate.

In Itamonte, Melastomataceae, Asteraceae and Myrtaceae are the most galled plant families. A similar result was found in Bertioga (restinga vegetation, São Paulo state) and Serra de São José (rupestrian fields, Minas Gerais). Melastomataceae and Asteraceae are also one of the most galled families in Espinhaço Range (cerrado, Minas Gerais), and Myrtaceae in Santa Rita do Passa-Quatro (cerrado, Minas Gerais) and in several restinga areas of the State of Rio de Janeiro. As these botanical families are well represented in the surveyed areas, the plant diversity hypothesis can explain this pattern.

Leaves were the most galled plant organ. This pattern has been observed in almost all Brazilian inventories, as well as in a majority of cases elsewhere in the world, probably because leaves represent an abundant and frequently renewable resource, with undifferentiated meristematic cells which are essential to gall growth (Maia 2011). Espinhaço Range represents an exception, as well as savanna areas in South 
Africa, where stem galls are the most frequent as indicated by Carneiro et al. (2009) and Veldtman \& McGeoch (2003), respectively. According to these authors, stems are safer than leaves as oviposition sites as they do not suffer mechanical injuries caused by winds or other herbivores. Furthermore, they can provide tissues with better food quality, due to their placement in relation to the vascular system. The high specificity of host plant organ is confirmed as almost all morphotypes occurred in a single plant organ.

Globose galls were the most frequent. The same shape predominance was observed in several localities and biomes: Espinhaço Range, MG (cerrado, Carneiro et al. 2009), Pernambuco (caatinga, Santos et al. 2001), Espírito Santo (restinga, Bregonci et al. 2010), and Goiás (cerrado, Araújo et al. 2007), suggesting that this is the most common gall shape in Brazil. The majority of the galls was glabrous and one-chambered as in all other Brazilian galls inventories, excepting by Delfinópolis, where hairy galls are more frequent than glabrous (54\% and 46\%, respectively) (Urso-Guimarães et al. 2003).

The galling guild of Itamonte comprises only three insect orders: Diptera, Hemiptera, and Lepidoptera. In other Brazilian inventories, galls of Coleoptera, Hymenoptera and Thysanoptera have been recorded in Barra de Maricá and Arraial do Cabo (RJ) (Monteiro et al. 1994), and Espinhaço Range (MG) (Carneiro et al. 2009); Coleoptera and Hymenoptera in Grumari (RJ) (Oliveira \& Maia 2005); Coleoptera in Boqueirão (MG) (Malves \& Frieiro-Costa 2012) and Pernambuco (Santos et al. 2011); Hymenoptera in Santa Rita do Passa Quatro (SP) (Urso-Guimarães \& ScareliSantos 2006); and Thysanoptera in Luiz Antônio (SP) (Saito \& Urso-Guimarães 2012). Hymenoptera and Thysanoptera are the less frequent galling insects in Brazil (Maia 2006 and Maia, in press.). Thysanopterous galls are rare throughout the world (Felt 1940), but Hymenopterous galls, especially those induced by Cynipidae are common, specially in the Holarctic region. There are more than 1,300 described galling species of Cynipidae (Ronquist \& Liljeblad 2001). The scarcity of galls induced by Hymenoptera and Thysanoptera appears to be a peculiar pattern of Brazil.

Cecidomyiidae (Diptera) was the most frequent galling insect, followed by Hemiptera and Lepidoptera. Cecidomyiidae are the most speciose and frequent galling insects in all zoogeographic regions (Gagné 2010). Hemiptera and Lepidoptera are also important gallers. In the Neotropical region, they are the second and third most frequent galling insects (Maia 2006). Coleoptera is the fourth (Maia 2012), but they are not found in the surveyed areas of Itamonte.

The associated fauna can be very rich, as pointed by Maia (2001), Maia \& Fernandes (2004) and Maia \& Azevedo (2009), but it showed a very low frequency and diversity in Itamonte: parasitoids were obtained from $6 \%$ of the gall morphotypes, successors from a single morphotype, predators and inquilines were not found. This is a surprising result as the frequency of parasitoids is higher than this value in all Brazilian inventories: 23\% in Luiz Antônio, SP (Saito \& Urso-Guimarães 2012); 31\% in Guarapari, ES (Bregonci et al. 2010); 35\% in Tiradentes, MG (Maia \& Fernandes, 2004); 48\% in Bertioga, SP (Maia et al. 2008); $60 \%$ in restingas of Rio de Janeiro (Maia \& Azevedo 2009), 71\% in Pirenópolis, GO (Araujo et al. 2007), excepting by Porto de Trombetas, where the frequency of parasitoids is about 3\% (Maia 2011). According to Fernandes \& Price (1992), parasitism is higher in mesic habitats because of longer maturation and hardening of the gall; however, this was not observed in the present study.

\section{Conclusion}

The results of the present study add evidences to support several known patterns or hypothesis, such as the plant diversity hypothesis, the predominance of leaf galls, the high specificity of host plant organ, the predominance of globoid, glabrous and one-chambered galls in Brazil, and the highest frequency of galling species of Cecidomyiidae (Diptera), Hemiptera and Lepidoptera in the Neotropical region. On the other hand, no evidence to the microenvironment hypothesis and to the prediction that parasitism is higher in mesic than in xeric habitats was provided.

\section{Acnowledgments}

Thanks to CNPq for financial support (Proc.), Dr. Bernardo Mascarenhas (Museu Nacional, UFRJ), Ms. Sueli Pereira (Museu Nacional, UFRJ) and Eduardo Barros (Museu Nacional, UFRJ) for field assistance, Lili Taylor and Solange Giordano de Freitas for logistic support.

\section{References}

ARAÚJO, W.S., GOMES-KLEIN, V.L. \& SANTOS, B.B. 2007. Galhas Entomógenas Associadas à Vegetação do Parque Estadual da Serra dos Pireneus, Pirenópolis, Goiás, Brasil. R. Bras. Bioci. 5(supl. 1):45-47.

BREGONCI, J.M., POLYCARPO, P.V. \& MAIA, V.C. 2010. Insect galls of the Parque Estadual Paulo César Vinha (Guarapari, ES, Brazil). Biota Neotrop. 10(1):265-274. http://www.biotaneotropica. org.br/v10n1/en/abstract?inventory+bn01410012010. http:// dx.doi.org/10.1590/S1676-06032010000100023

CARNEIRO, M.A.A., BORGES, R.A..X., ARAÚJO, A.P.A. \& FERNANDES, G.W. 2009. Insetos indutores de galhas da porção sul da Cadeia do Espinhaço, MG, Brasil. Rev. Bras. entomol . 53:570-592. http://dx.doi.org/10.1590/S0085-56262009000400007

COELHO, M.S, ALMADA, E., FERNANDES, G.W., CARNEIRO, M.A.A. SANTOS, R.M., QUINTINO, A.V. \& SANCHEZAZOFEIFA, A. 2009. Gall inducing arthropods from a seasonally dry tropical forest in Serra do Cipó, Brazil. Rev. Bras. entomol. 53:404-414. http://dx.doi.org/10.1590/S0085-56262009000300015

FELT, E.P. 1940. Plant Galls and Gall Makers. Comstock Publishing Co., Ithaca, N. Y., viii + 364p.

FERNANDES, G.W. 1992. Plant family size and age effects on insular gallforming species richness. Glob Ecol Biogeogr Let. 2:71-74. http://dx.doi.org/10.2307/2997508

FERNANDES, G.W. \& NEGREIROS, D. 2006. A comunidade de insetos galhadores da RPPN Fazenda Bulcão, Aimorés, Minas Gerais, Brasil. Lundiana 7(2):111-120.

FERNANDES G.W. \& PRICE P. 1992. The adaptive significance of the insect gall distribution: survivorship of species in xeric and mesic habitats. Oecologia 90:14-20. http://dx.doi.org/10.1007/ BF00317803

FERNANDES, G.W., TAMEIRÃO-NETO E. \& MARTINS, R.P. 1988. Ocorrência e caracterização de galhas entomógenas na vegetação do Campus Pampulha da Universidade Federal de Minas Gerais. Rev. Bras. Zool. 5(1):11-29. http://dx.doi.org/ 10.1590/S0101-81751988000100002

FERNANDES, G.W., CARNEIRO, M.A.A.,LARA, A.C.F., ALLAIN, L.R., JULIÃO, G.R., ANDRADE, G.I., REIS, T. R., \& SILVA, I.M. 1996. Galling insects on neotropical species of Baccharis (Asteraceae). Trop. Zool. 9:315-332. http://dx.doi.org/ 10.1080/03946975.1996.10539315 
FERNANDES, G.W., ARAÚ JO, R.C., ARAÚ JO, S.C., LOMBARDI, J.A., PAULA, A.S., LOYOLA-JÚNIOR, R. \& CORNELISSEN, T.G. 1997. Insect galls from savanna and rocky fields of the Jequitinhonha Valley, Minas Gerais, Brazil. Naturalia 22:221-244.

GAGNÉ, R.J. 1994. The gall midges of the Neotropical region. Ithaca: Cornell University Press. 352p.

GAGNÉ, R.J. 2010. A Catalog of the Cecidomyiidae (Diptera) of the world. http://www.ars.usda.gov/SP2UserFiles/Place/12754100/ Gagne_2010_World_Catalog_Cecidomyiidae.pdf. Captured on January

HONKALA, J. \& NIIRANEN, S. 2010. A birdwatching Guide to South-East Brazil. Portal do Bosque Association, 416p.

ITAMONTE. 2012. http://turismo.itamonte.mg.gov.br/meioambiente. asp. (05/xii/2012).

JULIÂO, G.R. 2007. Riqueza e abundância de insetos galhadores associados ao dossel de florestas de terra firme, várzea e igapó da Amazônia Central. Tese de Doutorado, Universidade Federal do Amazonas, Manaus, 144p.

JULIÃO G.R., AMARAL, M.E.C. \& FERNANDES, G W. 2002. Galhas de insetos e suas plantas hospedeiras do Pantanal sul-matogrossense. Naturalia 27:47-74.

MAIA, V.C. 2001. The gall midges (Diptera, Cecidomyiidae) from three restingas of Rio de Janeiro State, Brazil. Rev. Bras. Zool. 18(2):583-629. http://dx.doi.org/10.1590/S010181752001000200028

MAIA, V.C. 2006. Galls of Hemiptera, Lepidoptera and Thysanoptera from Central and South America. Pub. Avul. Mus. Nac. 110:3-22.

MAIA, V.C. 2011. Characterization of insect galls, gall makers, and associated fauna of Platô Bacaba (Porto de Trombetas, Pará, Brazil). Biota Neotrop. 11(4):37-53. http://www.biotaneotropica. org.br/v11n4/en/abstract?article+bn00511042011. http://dx.doi.org/ 10.1590/S1676-06032011000400003

MAIA, V.C. 2012. Coleopterous galls from the Neotropical region. Pap. Avulsos de Zool. 52(15):175-184.

MAIA, V.C. in press. Hymenopterous galls from South America. Pap. Avulsos de Zool..

MAIA, V.C. \& AZEVEDO, M.A.P. 2009. Micro-Hymenoptera associated with Cecidomyiidae (Diptera) galls at Restingas of the Rio de Janeiro State. Biota Neotrop. 9(2):151-164. http://www. biotaneotropica.org.br/v9n2/en/abstract?article+bn02809022009. http://dx.doi.org/10.1590/S1676-06032009000200015

MAIA, V.C. \& FERNANDES, G.W. 2004. Insect galls from Serra de São José (Tiradentes, MG, Brazil). Braz. J. Biol. 64(3):423-445. http://dx.doi.org/10.1590/S1519-69842004000300007

MAIA, V.C. \& OLIVEIRA, J.C. 2010. Insect galls of the Reserva Biológica Estadual da Praia do Sul (Ilha Grande, Angra dos Reis, RJ). Biota Neotrop. 10(4):227-237. http://www.biotaneotropica. org.br/v10n4/en/abstract?inventory+bn04110042010. http:// dx.doi.org/10.1590/S1676-06032010000400028

MAIA, V.C., AZEVEDO, M.A.P. \& COURI, M.S. 2002. New contribution to the knowledge of the gall midges (Diptera, Cecidomyiidae) from the restinga of Barra de Maricá (Rio de Janeiro, Brazil). Studia Dipt. 9:447-452.

MAIA, V.C., MAGENTA, M.A.G. \& MARTINS, S.E. 2008. Ocorrência e caracterização de galhas de insetos em áreas de restinga de Bertioga (São Paulo, Brasil). Biota Neotrop. 8(1):167197. http://www.biotaneotropica.org.br/v8n1/en/abstract?inventory+bn02408012008. Captured on January 2013. http://dx.doi.org/ 10.1590/S1676-06032008000100020

MALVES, K. \& FRIEIRO-COSTA, F.A. 2012. List of plants with galls induced by insects from the UNILAVRAS/Boqueirão Biological Reserve, Ingaí, State of Minas Gerais, Brazil. Check List 8(3):426-431.

MARTINELLI, G., BANDEIRA, J. \& BRAGANÇA, J O. 1989. Campos de Altitude. Index, Rio de Janeiro.

MONTEIRO, R.F., FERRAZ, F.F., MAIA, V.C. \& AZEVEDO, M.A.P. 1993. Galhas entomógenas em restinga: uma abordagem preliminar. In Anais do III Simpósio de Ecossistemas da Costa Brasileira, p. 210-220.

MONTEIRO, R.F., ODA, R.A.M., NARAHARA, K.L. \& CONSTANTINO, P.A.L. 2004. Galhas: diversidade, especificidade e distribuição. In C. F. D. Rocha, F. A. Esteves and F. R. Scarano (orgs). Pesquisas de longa duração na Restinga de Jurubatiba: ecologia, história natural e conservação. Rima, São Carlos, p. 127141.

ODA, R.A.M. 2006. Distribuição espacial de insetos fitófagos, com ênfase em galhadores, em três diferentes regiões do Brasil. Tese de Doutorado, Universidade Federal do Rio de Janeiro, Rio de Janeiro, 105p.

OLIVEIRA, J.C. \& MAIA, V.C. 2005. Ocorrência e caracterização de galhas de insetos na restinga de Grumari (Rio de Janeiro, RJ, Brasil). Arq. Mus. Nac. 63(4):669-676.

PRICE, P.W., WARING, G.L. \& FERNANDES, G.W. 1986. Hypotheses on the adaptive nature of galls. P. Entomol. Soc. Wash. 88:361-363.

RONQUIST, F. \& LILJEBLAD, J. 2001. Evolution of the gall wasphost plant association. Evolution 55(12):2503-2522.

RÜBSAAMEN, E.H. 1905. Beiträge zur Kenntnis aussereuropäischer Zoocecidien. II. Beitrag: Gallen aus Brasilien und Peru. Marcellia 4:65-85; 115-138.

RÜBSAAMEN, E.H. 1907. Beiträge zur Kenntnis aussereuropäischer Zoocecidien. III. Beitrag: Gallen aus Brasilien und Peru. Marcellia 6:110-173.

RÜBSAAMEN, E.H. 1908. Beiträge zur Kenntnis aussereuropäischer Zoocecidien. III. Beitrag: Gallen aus Brasilien und Peru. Marcellia 7:15-79.

RÜBSAAMEN, E.H. 1916. Beiträge zur Kenntnis aussereuropäischer Gallmücken. Sitzber. Ges. Natur. Freunde Berlin 1915:431-481.

SAITO, V.S. \& URSO-GUIMARÃES, M.V. 2012. Characterization of galls, insect galls and associated fauna of Ecological Station of Jataí (Luiz Antônio, SP). Biota Neotrop. 12(3):99-107. http://www. biotaneotropica.org.br/v12n3/en/abstract?article+bn02312032012. http://dx.doi.org/10.1590/S1676-06032012000300011

SANTOS, B.B., FERREIRA, H.D. \& ARAÚJO, W.S. 2010. Ocorrência e caracterização de galhas entomógenas em uma área de floresta estacional semidecídua em Goiânia, Goiás, Brasil. Acta Bot. Bras. 24(1):243-249. http://dx.doi.org/10.1590/S010233062010000100026

SANTOS, J.C; ALMEIDA-CORTEZ, J.S. \& FERNANDES, G.W. 2011. Richness of gall-inducing insects in the tropical dry forest (caatinga) of Pernambuco. Rev. Bras. entomol. 55(1):45-54. http:// dx.doi.org/10.1590/S0085-56262011000100009

TAVARES, J.S. 1909. Contributio prima ad cognitionem cecidologiae Braziliae. Brotéria (Zool.) 5:5-28.

TAVARES, J.S. 1917a. As cecídias do Brazil que se criam nas plantas da família das Melastomataceae. Brotéria (Zool.) 15:18-59.

TAVARES, J.S. 1917b. Cecídias brazileiras que se criam em plantas das famílias das Compositae, Rubiaceae, Lythraceae e Artocarpaceae. Brotéria (Zool.) 15:113-181.

TAVARES, J.S. 1918. Cecidologia brazileira. Cecídias que se criam nas plantas das famílias das Verbenaceae, Euphorbiaceae, Malvaceae, Anacardiaceae, Labiatae, Rosaceae, Anonaceae, Ampelidaceae, Bignoniaceae, Aristolochiaceae e Solanaceae. Brotéria (Zool.) 16:21-68.

TAVARES, J.S. 1920. Cecidologia brazileira. Cecídias que se criam em plantas das famílias das Leguminosae, Sapotaceae, Lauraceae, Myrtaceae, Punicaceae, Aurantiaceae, Malpighiaceae, Sapindaceae, Umbelliferae, Loranthaceae, Apocynaceae, Urticaceae, Salicaceae e Graminae. Brotéria (Zool.) 18:82-125.

TAVARES, J.S. 1921. Cecidologia brazileira. Cecidias que se criam em plantas das famílias das Leguminosae, Sapotaceae, Lauraceae, Myrtaceae, Punicaceae, Aurantiaceae,Malpighiaceae, Sapindaceae, Umbelliferae, Loranthaceae, Apocynaceae, Urticaceae, Salicaceae e Graminae. Brotéria (Série Zool.) 19:76-112, pl. I.

TAVARES, J.S. 1922. Cecidologia Brazileira. As restantes famílias. 
Brotéria (Zool.) 20:5-48.

TAVARES, J.S. 1925. Nova contribuição para o conhecimento da Cecidologia Brazileira. Brotéria (Zool.) 22:5-55.

URSO-GUIMARÃES, M.V. \& SCARELLI-SANTOS, C. 2006. Galls and gall makers in plants from the Pé-de-Gigante Cerrado Reserve, Santa Rita do Passa Quatro, SP, Brazil. Braz. J. Biol. 66:357-369.
URSO-GUIMARÃES, M.V., SCARELLI-SANTOS, C. \& BONIFÁCIO-SILVA, A.C. 2003. Occurrence and characterization of entomogen galls in plants from natural vegetation areas in Delfinópolis, MG, Brazil. Braz. J. Biol. 63(4):705-715.

ZIKAN, J F. \& ZIKAN, W. 1968. Inseto-fauna do Itatiaia e da Mantiqueira. III. Lepidoptera. Pesq. Agropec. Bras. 3:45-104. 